\title{
Deubiquitinating enzyme USP48 mediates pyroptosis and meliorates immune evasion in tumors by stabilizing GSDME expression
}

wang yunshan ( $\sim$ wangyunshansd@sdu.edu.cn )

The Second Hospital of Shandong University

\section{Yidan Ren}

The Second Hospital of Shandong University

\section{Maoxiao Feng}

The Second Hospital of Shandong University

\section{Xiaoyan Liu}

Department of Human Anatomy and Key Laboratory of Experimental Teratology

\section{Xiaodong Hao}

The Second Hospital of Shandong University

\section{Juan Li}

Department of Clinical Laboratory, The Second Hospital of Shandong University

\section{Peilong Li}

The Second Hospital, Cheeloo College of Medicine, Shandong University https://orcid.org/0000-0002-

7441-6514

Jie Gao

Shandong University

Qiuchen Qi

Shandong University

Lutao Du

Shandong University

Chuanxin Wang

The Second Hospital, Cheeloo College of Medicine, Shandong University https://orcid.org/0000-00023796-6293

\section{Article}

Keywords: Pyroptosis, tumors, ubiquitin-related proteins

Posted Date: November 11th, 2021

DOI: https://doi.org/10.21203/rs.3.rs-1046804/v1 
License: (c) (i) This work is licensed under a Creative Commons Attribution 4.0 International License. Read Full License 


\title{
Deubiquitinating enzyme USP48 mediates pyroptosis and meliorates immune evasion in tumors by stabilizing GSDME expression
}

\begin{abstract}
Pyroptosis is a kind of programmed cell death, which is characterized by the activation of inflammatory caspase and the cleavage of gasdermin proteins. It is widely involved in the occurrence and development of tumors. Studies have shown that ubiquitin related proteins play a key regulatory role in many biological processes. However, the role and molecular mechanism of ubiquitin-related proteins in pyroptosis have not been well identified. Here, using CRISPR-Cas9 screening, we identified a deubiquitinating enzyme (USP48) that has the most significant regulatory effect on cell pyroptosis. USP48 stabilizes GsderminE (GSDME) expression by causing deubiquitination of it, thereby achieving its regulatory effect on pyroptosis. USP48 prevents the degradation of GSDME by inhibiting K63-linked ubiquitination at positions K120 and K189 of GSDME. Clinical tissue testing confirmed that the expression of USP48 has a significant positive correlation with GSDME and pyroptosis-related factors. GSDME plays a crucial role in the regulation of cell pyroptosis by USP48. The single-cell sequencing results showed that the functions of $\mathrm{T}$ cells and tumor-associated macrophages in the tumor microenvironment are inhibited to varying degrees after USP48 gene knockout. Finally, the tumor formation experiment in mice confirmed that overexpression of USP48 could effectively improve the therapeutic effect of PD-1 inhibitors. These findings define a pyroptosis regulation pathway and indicate that activation of the pharmacological activity of USP48 may provide an effective strategy to sensitize cancer cells to pyroptosis-related immunotherapeutic resistance.
\end{abstract}

\section{Introduction}

Pyroptosis is a type of programmed cell death mediated by the gasdermin family of proteins, which has been discovered in recent years ${ }^{1}$. Different proteases or granzymes mediate the cleavage of the gasdermin family proteins to release the N-terminal domain, which is then transported to the cell membrane to form multimeric pores, causing changes in membrane permeability, and leads to cell swelling and membrane rupture ${ }^{2-4}$. Pyroptosis is an important immune response of the body, which plays an important role in antagonizing infection and endogenous danger signals ${ }^{5}$. It is widely involved in the occurrence and development of tumors, infectious diseases, metabolic diseases, nervous system-related diseases and atherosclerotic diseases ${ }^{6}$.

Recent studies have found that gasdermin E (GSDME, also known as DFNA5) is mutated in familial aging-related hearing loss. GSDME can be cleaved by caspase 3, thereby converting noninflammatory apoptosis into pyroptosis in GSDME-expressing cells ${ }^{7}$. In many cancers, the expression of GSDME is suppressed ${ }^{8}$. Studies have shown that reduced GSDME levels are associated with reduced survival rates caused by breast cancer, which suggests that GSDME may be a tumor suppressor ${ }^{9}$. The evidence that GSDME may be used as a tumor suppressor also includes 
promoter DNA methylation in many primary cancers that can inactivate GSDME; GSDME inhibits colony formation, proliferation and invasiveness of gastric cancer, melanoma, and colorectal cancer cells; and the decreased expression of GSDME is closely related to worse 5-year survival rates and poor metastasis of many patients with cancer ${ }^{10-13}$. Recent studies have revealed that the expression of GSDME enhances the phagocytosis of tumor-associated macrophages on tumor cells, as well as the number and function of tumor-infiltrating natural killer cells and $\mathrm{CD} 8^{+} \mathrm{T}$ lymphocytes. Granzyme B from natural killer cells can also activate caspase-independent pyroptosis in target cells by directly cleaving GSDME at the same site as caspase $3{ }^{14,15}$. Therefore, GSDME in tumor tissues acts as a tumor suppressor by activating pyroptosis and enhancing anti-tumor immunity.

Ubiquitin is an evolutionarily conserved $8.5 \mathrm{kDa}$ protein, which is covalently linked to the $\mathrm{N}$ terminal or lysine residue of the substrate protein through the sequence reaction of ubiquitin activating enzyme (E1), ubiquitin coupling enzyme (E2) and ubiquitin ligase (E3) ${ }^{16}$. The ubiquitin chain can be formed by connecting ubiquitin with lysine 48 of ubiquitin itself, resulting in the degradation of substrates by proteasomes. Other types of polyubiquitin chain ligation and monoubiquitination can lead to degradation or non-degradable effects on substrates, such as repositioning, promoting protein-protein interactions and regulating signal events ${ }^{17}$. Ubiquitination is regulated by more than 1000 human proteins, accounting for about $4 \%$ of the proteome, including two E1s, about $40 \mathrm{E} 2 \mathrm{~s}$, more than $600 \mathrm{E} 3 \mathrm{~s}$ and about 100 deubiquitinases (DUBs) ${ }^{18}$. It has been reported that there are more than 8000 ubiquitination sites on thousands of proteins ${ }^{19}$. Studies have shown that ubiquitin-related proteins play a key regulatory role in many biological processes, such as the cell cycle, DNA damage, apoptosis and autophagy ${ }^{20-23}$. However, the roles and molecular mechanisms of ubiquitin-related proteins in pyroptosis have not been well identified.

The latest progress of CRISPR-Cas9 gene screening technology makes it possible to measure gene importance, cancer cell dependence, and genetic interactions in human cells in a high-throughput manner ${ }^{24}$. Here, we screened and identified USP48, a ubiquitinase that plays an important role in regulating cell pyroptosis by applying the CRISPR-Cas9 gene screening technology, combined with the detection of related secretory factors. In terms of molecular mechanism, USP48 stabilizes its expression by causing deubiquitination of GSDME, achieving its regulatory effect on pyroptosis. Clinical tumor tissue testing confirmed that the expression of USP48 has a significant positive correlation with GSDME and pyroptosis-related factors. The single-cell sequencing results showed that the immune microenvironment in the tumor tissues of the mice after USP48 gene knockout was significantly changed. Consistent with recent reports, the functions of $\mathrm{T}$ cells and tumor-associated macrophages in the tumor microenvironment are inhibited to varying degrees after pyroptosis. Finally, the tumor formation experiment in mice confirmed that overexpression of USP48 could effectively improve the therapeutic effect of PD-1 inhibitors.

\section{Results}

\section{USP48 is involved in the regulation of pyroptosis}


Pyroptosis is a newly identified pattern of programmed cell death, which is mainly mediated by inflammasomes through the activation of a variety of caspases (including caspase-1), resulting in the shear and multimerization of a variety of gasdermin family members( including GSDMD), which caused cell perforation and cell death ${ }^{25}$. In order to find related genes involved in the regulation of pyroptosis, we conducted CRISPR-Cas9 gene screening in 293T cells, using the secretion of LDH after Raptinal (a fast caspase-3 activator) treatment as a readout, and the obtained genes were further screened and verified by single siRNA screening technology (Figure 1A). The screening library consists of 384 unique sgRNAs, knocking down the expression of 96 related genes. According to the fold change of LDH secretion after sgRNA transfection, 39 genes were selected as the main genes. Among them, the silencing of 18 candidate genes increased the secretion of LDH by more than 2-fold, while the silencing of the other 21 genes inhibited the secretion of LDH (Figure 1B). In order to further screen the obtained genes, we designed four independent small interfering RNAs (siRNAs) for each gene. The results showed that the interference of USP48 had the most significant inhibitory effect on the release of LDH in cells (Figure 1C).

Ubiquitination is an important item in post-translation. Its most well-known function is to guide the degradation of proteins. Ubiquitination is reversible and can be reversed by a large group of proteases called deubiquitinases (DUBs) ${ }^{26}$. USP48 is an important member of the USP family of DUBs. In order to further clarify the role of USP48 in pyroptosis, we silenced the expression of USP48 by transfecting siRNA into the $293 \mathrm{~T}$ cell line (Figure 1D) and treated the cells with Raptinal to detect the effect of USP48 silencing on LDH, IL-1 $\beta$, and IL-18 in 293T cells (Figure 1G). The results showed that the silence of USP48 could significantly inhibit the release of LDH, IL-1 $\beta$ and IL-18 in 293T cells. In addition, we also observed the morphological changes and the absorption of SYTOX green in 293T cells transfected with siRNA (si-NC) or USP48-specific siRNA (si-USP48) after Raptinal treatment by time-lapse microscopy, and the results showed that USP48 silencing significantly inhibited Raptinal-induced cell membrane rupture and SYTOX green absorption (Figure 1F-I). The above results were further confirmed in L929 cells, which is a mouse fibroblast cell line (Supplementary Figure 1A-F). In addition, we also found that USP48 silencing inhibited the Raptinal-induced release of HMGB1 (Figure 1J, Supplementary Figure 1G). In addition, upregulating the expression of USP48 in 293T cells also obtained consistent results with the above (Supplementary Figure 1H-K). In summary, we have preliminarily confirmed that USP48 may be involved in the regulation of pyroptosis.

\section{USP48 regulates the expression of GSDME}

In order to further clarify the specific mechanism of USP48 regulating cell pyroptosis, we performed proteomic sequencing in $293 \mathrm{~T}$ cells overexpressing USP48 or transfected with an empty plasmid (Figure 2A). It was found that the expressions of a total of 112 proteins were upregulated, and the expressions of 258 proteins were negatively correlated with the overexpression of USP48 
1 (Supplementary Figure 2A and B, Supplementary Table 2). In addition, the results also showed that

2 USP48 was involved in the regulation of various cell biological processes, including pyroptosis and

3 cell metabolism (Supplementary Figure $2 \mathrm{C}$ and D). To further identify the proteins physically associated with USP48, we used anti-Flag affinity purification mass spectrometry to identify potential USP48-interacting proteins in 293T cells expressing Flag-tagged USP48 and found 59 proteins that could directly interact with USP48 (Supplementary Table 3). After combined analyses of both mass spectrometry and proteomics results, we obtained eight related proteins (Figure 2B and C). In order to further verify the above results, we tested the changes in the expression levels of these eight proteins in 293T cells overexpressing USP48 and knocking down USP48 and found that the changes in GSDME were the most significant (Figure 2D and E).

GSDME is a newly discovered important protein involved in the regulation of pyroptosis and an important tumor suppressor. In cells expressing GSDME, GSDME can be cleaved and activated by caspase-3, resulting in cell pyroptosis. Subsequently, we further confirmed the physical interaction between USP48 and GSDME by CO-IP (Figure 2F and G). Subsequently, we confirmed the regulatory effect of USP48 on GSDME through immunohistochemistry and Western blotting in human pancreatic cancer and paraneoplastic tissues, as well as human liver cancer and paraneoplastic tissues, and confirmed the positive correlation between USP48 and GSDME (Supplementary Figure 3A-F). In addition, we also found that USP48 did not affect the cleavage of GSDME (Figure 2H). In summary, we found that USP48 could regulate the expression of GSDME (but does not affect its cleavage) and can be directly combined with GSDME.

\section{USP48 affects pyroptosis by regulating the expression of GSDME}

Due to the key role of GSDME in activating cell pyroptosis, we speculate that GSDME also plays an important role in the regulation of cell pyroptosis by USP48. To confirm this hypothesis, we established a 293T cell line that simultaneously expressed USP48 and shRNA targeting GSDME (Figure 3A). As expected, the downregulation of GSDME substantially rescued the promotion of overexpression of USP48 on the production of LDH (Figure 3B), IL-18 (Figure 3C), and IL-1 $\beta$ (Figure 3D) in 293T cells after treatment with Raptinal. Conversely, in 293T cell lines expressing both shUSP48 and GSDME (Figure 3E), it was also confirmed that overexpression of GSDME could rescue the inhibitory effect of USP48 knockdown on the production of LDH (Figure 3F), IL$1 \beta$ (Figure 3G), and IL-18 (Figure 3H) in 293T cells after treatment with Raptinal. Subsequently, we also used time-lapse microscopy to observe the morphology and absorption of SYTOX green in the 293T cell line expressing USP48 and shRNA targeting GSDME after treatment with Raptinal and consistent results were obtained (Figure 4I and J). In addition, we also obtained results consistent with the above in L929 cells (Supplementary Figure 4). Interestingly, although the study confirmed that GSDME can be cleaved by caspase-3 and induce pyroptosis, our results suggest that USP48 did not achieve regulatory effects on GSDME, as well as pyroptosis, through caspase-3. Activating or overexpressing caspase-3 in 293T cells with USP48 knockdown, respectively, did not 
affect the ability of USP48 to regulate GSDME and pyroptosis levels (Supplementary Figure 5). In conclusion, we confirmed that USP48 may promote the expression of GSDME in a way that was independent of caspase-3, thereby promoting the occurrence of pyroptosis.

\section{USP48 prevents the degradation of GSDME by deubiquitinating it}

The above results have preliminarily confirmed that USP48 realizes its regulation through physical interaction with GSDME, but the specific mechanism is still unclear. USP48 is an important member of the deubiquitinating enzyme family. Studies have confirmed that it is involved in mediating Gli1, TRAF2, Mdm2 and many other proteins ${ }^{27-30}$. Therefore, we speculate that USP48 deubiquitinates GSDME, thereby inhibiting the proteasome degradation of GSDME. The ubiquitin-proteasome pathway is an important protein degradation regulatory system in cells.

In order to further confirm whether USP48 affects the stability of GSDME, we found that the proteasome-specific inhibitor MG132 could effectively reverse the impact of USP48 knockdown on GSDME (Figure 4A) and through cycloheximide (CHX) chase analysis to evaluate the potential of USP48 in regulating GSDME protein turnover rate, which shows that the reduction of the USP48 level is obviously related to the reduction of the half-life of GSDME (Figure 4B). Interestingly, we found that the change of USP48 expression had no effect on GSDME at the RNA level (Supplementary Figure 6A and B), which was also consistent with USP48 affecting the protein stability of GSDME. In view of the above observations, we established 293T cells with Dox-induced wild-type USP48 (USP48/WT) and catalytically inactive mutant USP48 (USP48/C98A). It was found that only wild-type USP48 gradually increased GSDME levels in a Dox dose-dependent manner (Figure 4C), while no significant changes in GSDME protein levels were detected in cells expressing USP48/C98A (Figure 4D). Through further experiments, we found that the overexpression of wild-type USP48 significantly reduced the ubiquitination level of GSDME, while 293 T cells expressing USP48/C98A had no effect on the ubiquitination of GSDME (Figure 4E). In contrast, knockdown of USP48 in 293T cells led to the accumulation of ubiquitinated GSDME (Figure 4F). The above results were further confirmed in L929 cells (Supplementary Figure 6C-E). Besides, we also found that overexpression of USP48/C98A in 293T cells had no effect on pyroptosis (Supplementary Figure 6F-H).

Ubiquitin can post-translationally modify proteins. The binding of a single ubiquitin molecule to a target protein is referred to as monoubiquitination, and additional ubiquitin portions can be spliced to that initial ubiquitin to form polyubiquitin (polyUb) chains. These polyUb chains can be linked by all the lysines present in ubiquitin (K6, K11, K27, K29, K33, K48 and K63). Two of the most fully characterized forms of polyubiquitination occur by attachment to lysine 48 (K48) or lysine 63 $(\mathrm{K} 63)^{31}$. We also found that USP48 affects K48-linked ubiquitin in GSDME, but it has no effect on K63-linked ubiquitination (Figure 4G). Then, we predicted the ubiquitination sites of GSDME through the website (http://plmd.biocuckoo.org/index.php) and verified the seven predicted sites 
(K30, K39, K120, K161, K189, K240, and K248) through in vitro ubiquitination experiments. To examine the K48-linked ubiquitination sites, HEK293T cells were co-transfected with the seven Myc tagged mutants of GSDME and HA-ubiquitin K48. An in vitro ubiquitination assay showed that ubiquitin K48 ubiquitinated GSDME at the K30, K120 and K189 sites (Figure 4H and I). Next, in order to identify the target residues of GSDME modified by USP48, we constructed corresponding point mutants at positions K30, K120 and K189 of GSDME (Figure 4J), HEK293T cells were co-transfected with the GSDME mutant labeled with Myc, HA-ubiquitin K48, and FlagUSP48. The results showed that USP48 inhibited the K48-linked ubiquitination of GSDME at K120 (Figure 4K). Taken together, these results indicate that USP48 prevents the degradation of GSDME by inhibiting K48-linked ubiquitination at position K120 of GSDME.

\section{USP48 affects anti-tumor immunity by regulating the expression of GSDME}

Our above results have preliminarily confirmed that USP48 can promote the expression of GSDME, thereby promoting the occurrence of pyroptosis in cells. In order to further clarify this result, we

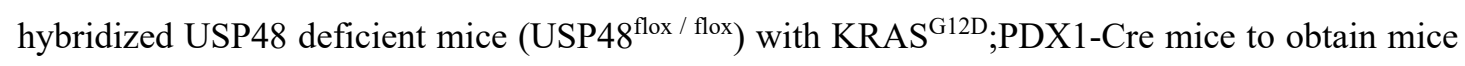
models with specific deletion of USP48 in the pancreas. The expression of USP48 and GSDME in the tissues was then detected by immunohistochemistry and immunofluorescence using pancreatic tissue from the above mice and laboratory-preserved liver tissue from mice lacking USP48 specificity ${ }^{32}$, and the results showed that the expression levels of USP48 were positively correlated with the expression of GSDME (Figure 5A-F). In addition, compared with $\mathrm{Kras}^{\mathrm{G} 12 \mathrm{D}}$;PDX1-Cre (KC) mice, the loss of USP48 significantly promoted the occurrence of pancreatic tumors. $\mathrm{Kras}^{\mathrm{G} 12 \mathrm{D}}$;USP48 $8^{\text {flox/flox}}$;PDX1-Cre (KUC) mice had already developed acinar ductal metaplasia (ADM) at about 3 months and obvious cancer occurred in about 5 months. The deletion of USP48 also significantly reduced the survival time of mice (Supplementary Figure 7). Western blotting was used to detect the expression of USP48 and GSDME in the liver and pancreas of the above mice for further detection and consistent results were obtained (Figure 5G and $\mathrm{H}$ ). The above results further confirmed the regulatory effect of USP48 on GSDME in vivo.

Pyroptosis is a pro-inflammatory form of cell death. Unlike apoptosis, pyroptosis is a pathological death in which cells swell until their membranes rupture, resulting in leakage of cytoplasmic content and a strong inflammatory immune response ${ }^{33}$. Previous studies have shown that GSDME inhibits tumor growth in mice by promoting cell anti-tumor immunity. Compared with control mice, mice expressing GSDME had more natural killer cells and CD8 $8^{+}$cytotoxic $\mathrm{T}$ killer cells in tumors and expressed more toxic proteins and cytokines ${ }^{14}$. Therefore, we speculate that USP48 may exert its anti-tumor effect by promoting the expression of GSDME and then promoting the anti-tumor immune response of cells.

In order to confirm this hypothesis, we explored the effect of the loss of USP48 on the immune cell population in PDAC tissues. The immunofluorescence results showed that the tumor-infiltrating 
$\mathrm{CD} 8+$ and CD4 $+\mathrm{T}$ cells were significantly reduced in KUC mice (Figure 5I-J). The proportion of natural killer cells and CD8+ cytotoxic T killer cells was significantly reduced, but the proportion of tumor-associated macrophages (TAMs) and Treg cells was significantly increased (Figure 5K). Together, these data imply that USP48 can affect the anti-tumor immunity of cells by regulating the expression of GSDME.

\section{Single-cell sequencing experiments clarify the regulatory effect of USP48 on anti-tumor immunity}

In order to further clarify the anti-tumor immune function of USP48, we performed single-cell sequencing in pancreatic tissues of KC and KUC mice. Single-cell RNA sequencing was performed using the 10x chromium method. Consistent with the enrichment approach, all cells were positive for the CD45 gene (PTPRC) irrespective of treatment (Figure 6A). We applied the size clustering algorithm as a quality control indicator for cells. Figure 6B shows the cell distribution in pancreatic tumor tissues of $\mathrm{KC}$ and KUC mice. Then, we applied the size clustering algorithm as the quality control index of the cells, divided the cells into groups, and defined five cell populations that capture the TAMs, dendritic cells (DCs), T cells, monocyte-1 cells and monocyte-2 cells (Figure 6B and C). By comparing the differences of cell subgroups in $\mathrm{KC}$ and $\mathrm{KUC}$ pancreatic cancer tissues, we found that there were significant differences in the number of cells in TAMs and monocyte- 2 cells. Specifically, the downregulation of USP48 expression significantly increased the number of TAMs and monocyte-2 cells (Figure 6D). We showed the difference of marker genes in each cell subgroup through heat maps and dot maps $(\mathrm{Cd} 3 \mathrm{~d}=\mathrm{T}$ cells; $\mathrm{Cd} 14=$ monocytes; $\mathrm{C} 1 \mathrm{qb}=\mathrm{TAMs}$; $\mathrm{S} 100 \mathrm{a} 3$ and Thbs 1 = monocytes; and $\mathrm{Clec} 9 \mathrm{a}=\mathrm{DCs}$ ) (Figure $6 \mathrm{E}$ and $\mathrm{F}$ ) and obtained the number and proportion of each cell subgroup in KC and KUC pancreatic cancer tissues (Figure 6G).

In order to further determine the immune cell subpopulations regulated by USP48, we first performed a subpopulation analysis of T cells in KC and KUC pancreatic cancer tissues (Figure 7A). It was found that the proportion of exhaustible $\mathrm{T}$ (Tex) cells and Treg cells in KUC pancreatic cancer tissues increased significantly, indicating that the reduction of USP48 expression inhibited the occurrence of tumor immunity (Figure 7B). We also used UMAP to show the expression of standard marker genes for $\mathrm{T}$ cell subgroup classification and the ratio of $\mathrm{T}$ cell subgroups in $\mathrm{KC}$ and KUC pancreatic cancer tissues, further confirming the above results. (Figure 7C and D). Subsequently, we used multicolor immunofluorescence experiments to detect the distribution of Tex cells and Treg cells in pancreatic cancer tissues of $\mathrm{KC}$ and KUC mice, and the results obtained were consistent with the sequencing results (Figure 7E and F).

In the above results, we have initially found that knockdown of USP48 significantly increased the proportion of TAM subgroups (Figure $7 \mathrm{G}$ and $\mathrm{H}$ ). Therefore, further analysis of TAMs was performed, and the same results were obtained. Multicolor immunofluorescence experiments also 
confirmed that the lack of USP48 increased the distribution of TAMs in pancreatic cancer tissues (Figure 7I) . In summary, we applied 10x single-cell sequencing technology to confirm that USP48 deletion inhibited the anti-tumor immunity of pancreatic cancer cells.

\section{USP48-GSDME modulates the sensitivity of mice to anti-PD-1 immunotherapy}

In order to further clarify the role of USP48 in anti-tumor immunity, Pan02 cells overexpressing USP48 or empty vectors were subcutaneously implanted into C57 mice. The mice were treated with aPD-1 (an anti-PD-1 antibody) on days 7, 11 and 15, and the growth of the mice was continuously observed and killed on day 30 (Figure 8A). PD-1 antibody is an important immunotherapy. It has a good therapeutic effect in the treatment of more than 10 malignant tumors, such as lung cancer, lymphoma, liver cancer, gastric cancer and pancreatic cancer ${ }^{34}$. The results showed that, compared with the empty vector, overexpression of USP48 significantly inhibited the growth of tumors in mice. In addition, overexpression of USP48 also significantly improved the sensitivity of mice to anti-PD-1 treatment (Figure 8B-E). To further clarify its mechanism, we expressed shGSDME or scramble in Pan02 cells overexpressing USP48 and injected them subcutaneously into C57 mice. Consistent with the expected results, knocking down the expression of GSDME in Pan02 mice overexpressing USP48 can effectively reverse the inhibitory effect of USP48 overexpression on tumor growth and the promotion of anti-PD-1 treatment sensitivity (Figure 8F-I).

Subsequently, we performed flow cytometry in mouse tumors, and the results were consistent with the previous results, namely, overexpression of USP48 can reduce the proportion of TAMs in mouse tumor tissues, while increasing the level of NK cells and knocking down GSDME can reverse this result (Figure 8G, Supplementary Figure 8). These results further confirmed the important role of the USP48-GSMDE pathway in anti-tumor immunity and discovered its key regulatory role in tumor immunotherapy.

\section{Discussion}

Protein ubiquitination is a strictly controlled process and is reversible. It can be reversed by a large group of proteases called deubiquitinating enzymes (DUBs). Most deubiquitinating enzymes can break down and release ubiquitin from the substrate protein, edit the ubiquitin chain and process ubiquitin precursors, and some deubiquitinases are related to editing or processing ubiquitin-like proteins and binding proteins ${ }^{35}$. USP48 is a deubiquitinating enzyme expressed in almost all human tissues. It has been shown that USP48 has a key role in the development and progression of numerous diseases. Cetkovska et al. found a stabilizing effect of USP48 on Mdm2 in osteosarcoma cells, U2OS and H1299 lung cancer cells, and overexpression of Mdm2 was associated with loss of p53 tumor suppressor activity in several human cancers ${ }^{29}$. In addition, in glioblastoma, USP48 can activate Gli-dependent transcription by stabilizing the Gli1 protein, with implications for cell proliferation and tumorigenesis ${ }^{27}$. Our previous study also demonstrated that USP48 can play a key regulatory role in hepatocarcinogenesis and development of liver cancer by regulating the stability 
of SIRT6 ${ }^{32}$. However, the regulation of USP48 in pyroptosis and anti-tumor immunity has not been investigated.

Pyroptosis, a type of non-classical apoptotic pathway, is a mechanism of programmed necrosis in inflammatory cells ${ }^{36}$. The concept of pyroptosis was first proposed by Cookson and Brennan in 2001 to describe a caspase-1-dependent mode of cell death in inflammatory cells ${ }^{37}$. However, for a long period of time, the mechanism of pyroptosis has not made great progress. Until 2015, Academician Shao Feng from the Beijing Institute of Life Sciences reported that the process of pyroptosis would be accompanied by the cleavage of GSDMD by caspase, and the cleaved GSDMD would form holes in the cell membrane, leading to the release of IL-1 $\beta$, IL-18 and other inflammatory factors ${ }^{25}$. Up to this point, the mechanism of pyroptosis had been studied clearly. In this study, we applied CRISPR-Cas9 high-throughput screening technology and found that USP48 had a significant regulatory effect on pyroptosis.

GSDME is also an important member of the gasdermin family. Previously, it was shown that the promoter of GSDME was methylated in a variety of cancer cells, and this epistatic modification inhibited its expression in cancer cells. In addition, it was also found that GSDME could inhibit the development of a variety of cancers, including breast cancer and melanoma ${ }^{38,39}$. In 2017, Shao Feng et al. revealed its key role in pyroptosis and found that GSDME could be cleaved and activated by caspase- 3 , which in turn converts apoptosis into pyroptosis ${ }^{7}$. Interestingly, our results found that USP48 can affect the expression of GSDME (and thus pyroptosis) through physical interaction with GSDME and does not affect the activation and cleavage of GSDME by caspase-3.

Our study revealed the regulatory role of USP48 on pyroptosis and its molecular mechanism, and we found that USP48 promotes pyroptosis by deubiquitinating GSDME, inhibiting its ubiquitinated degradation, promoting its expression, and ultimately promoting the development of pyroptosis. Unlike immunosilencing apoptosis, pyroptosis is characterized by cell membrane rupture in which numerous cytokines and danger signaling molecules are released, activating the immune system and leading to an inflammatory response ${ }^{40}$. In 2020, Judy Lieberman's group at Harvard Medical School and Boston Children's Hospital found that granzyme B from natural killer cells can directly cleave GSDME and activate pyroptosis, which occurs to further activate the anti-tumor immune response and inhibit tumor growth ${ }^{14}$. Given the important role of pyroptosis in anti-tumor immunity, we speculate that USP48 also has a regulatory role on cellular anti-tumor immunity, which was confirmed by both single-cell sequencing technology and flow cytometry. We found that USP48 could regulate the ratio and number of TAMs, NK cells, Tregs and other immune cells.

The tumor immune microenvironment is a highly complex system. It has also become an important research hotspot in recent years. With more and more in-depth research on immunotherapy and the continuous improvement of immunotherapy efficacy, immunotherapy has begun to be well-known 
to the public. PD-1 inhibitors are a new class of drugs that block PD-1 and are used to treat certain types of cancer by activating the immune system to attack tumors ${ }^{41,42}$. We found that changes in USP48 expression significantly modulated the efficacy of PD-1 inhibitors by constructing a xenograft tumor model in C57 mice, and increased USP48 expression significantly improved the therapeutic effect of PD-1 inhibitors in mouse tumors.

In conclusion, our study identified the key regulatory role of USP48 on pyroptosis and elucidated its molecular mechanism, in addition to the role of USP48 in anti-tumor immunity and immunotherapy. Therefore, specifically targeting the USP48 or USP48-GSDME axis may be a potential future therapeutic strategy. Nevertheless, our study leaves much to be desired, as we have only described the regulatory role of USP48 in anti-tumor immunity and immunotherapy. But, the specific molecular mechanisms need to be further elucidated.

\section{Materials and Methods}

\section{Cell cultures}

HEK293T, L929 and Pan02 cell lines were purchased from ATCC. All three cells were subcultured using DMEM (Gibco), containing 10\% fetal bovine serum (Gibco), in a cell incubator at $37^{\circ} \mathrm{C}$ and $5 \% \mathrm{CO}_{2}$ saturated humidity.

\section{Retroviral infection and overexpression of USP48 and GSDME}

The siRNA used for the knockdown of USP48 and GSDME in the cells was purchased from GenePharma (Shanghai, China). The siRNAs were transfected into cells using Lipofectamine 2000 (Invitrogen) according to the manufacturer's instructions. Lentivirus-mediated overexpression and knockdown of USP48 and GSDME against cells was purchased from GeneChem (Shanghai, China). Cells ( $30 \%$ confluence) were incubated in medium containing optimal dilutions of lentivirus mixed with polybrene. After 48 hours of transfection, cells were subjected to puromycin selection (5 $\mathrm{mg} / \mathrm{mL}$ ) to obtain stably transfected cells.

\section{Tissue specimens}

The collection of adjacent normal tissue specimens from a standard distance $(3 \mathrm{~cm})$ from the edge of the tumor tissue removed from patients with PDAC or HCC undergoing surgical resection was performed. All clinical specimens used in this study were histopathologically and clinically diagnosed. In order to use these clinical data for research purposes, the consent and approval of the Institutional Research Ethics Committee of the Second Hospital of Shandong University was obtained in advance. The research complied with all relevant ethical norms involving human participants.

\section{Animals and animal model}


1 Alb-Cre mice and Kras ${ }^{\text {G12D; }}$;DD-Cre mice were crossed with USP48 $8^{\text {flox/flox }}$ mice to obtain liver- or pancreas-specific knockout mice. All animal experiment procedures followed the guidelines of the

3 National Institutes of Health and the guidelines of the Second Hospital of Shandong University. The USP4 $8^{\text {flox/flox }}$ mice were generated by Cyagen Biosciences Inc. (Guangzhou, China).

\section{CRISPR Knockout Pooled Lentiviral sgRNA Libraries}

A combined library of thousands of defined single-guide RNA (sgRNA or gRNA) sequences can disrupt (or "knock out") hundreds of genes in an entire population of cells in a single experiment. The cell population is then screened for the release of lactate dehydrogenase, so the specific gene driving that phenotype can be identified. The CRISPR Knockout Pooled Lentiviral sgRNA Library was purchased from Dharmacon.

\section{Immunohistochemistry}

The specimens were fixed with formalin, paraffin-embedded sections, and the protein to be detected was evaluated by immunohistochemical staining. Two independent observers scored the proportion of positively stained tumor cells and the staining intensity. The cut-off values for high and low expression of the protein of interest are selected based on the measurement of heterogeneity using the log-rank test on overall survival.

\section{Multiplex immunofluorescence}

Tissues were fixed overnight in $4 \%$ PFA at $4{ }^{\circ} \mathrm{C}$ and embedded in paraffin. After preparation into sections, rehydration was performed through an ethanol series. Antigen retrieval was performed using a microwave oven in $10 \mathrm{mM}$ sodium citrate $(\mathrm{pH}$ 6). Sections were blocked with $10 \%$ goat serum and incubated with primary antibody $(1: 200)$ at $4{ }^{\circ} \mathrm{C}$ overnight. Then, the sections were thoroughly washed and incubated with secondary antibody (1:1000) and DAPI for 1 hour at room temperature. Sections were thoroughly washed and mounted in an anti-fade fluorescent mounting medium (Abcam; ab104135).

\section{Western Blotting and co-immunoprecipitation (co-IP)}

The cells were treated with RIPA buffer containing 1x protease inhibitor and phosphatase inhibitor, and the product was collected. The expression of corresponding proteins was detected by SDSPAGE gel electrophoresis, and Western blotting and band quantification were performed using the ChemiDoc $^{\text {TM }}$ MP Imaging System. For co-IP, the cell lysate $(1 \mathrm{~mL})$ was mixed with $10 \mu \mathrm{L}$ of antiMYC, anti-Flag or anti-HA antibody and $20 \mu \mathrm{L}$ of protein G magnetic beads (MCE) at $4{ }^{\circ} \mathrm{C}$ overnight. Then, the IP beads were washed three times with lysis buffer and then heated in SDS loading buffer at $100^{\circ} \mathrm{C}$ for 5 minutes. The product was used for SDS-polyacrylamide gel electrophoresis and Western blot analysis.

\section{In vitro ubiquitination assay}


USP48 and GSDME proteins were expressed using a TNT fast coupled transcription/translation system (Promega, Madison, Wisconsin, USA). Use A ubiquitination kit (Boston Biochem, Minnesota, USA) was used to perform ubiquitination analysis according to the manufacturer's recommended protocol.

\section{Quantitative Real-time PCR (qRT-PCR)}

Total RNA was isolated from cells cultured with TRIzol reagent (Invitrogen) as directed. According to the manufacturer's instructions for the reagent, the PrimeScript ${ }^{\mathrm{TM}}$ RT Reverse Transcription Kit (TaKaRa) was used to reverse the RNA $(1 \mu \mathrm{g})$ to cDNA $(20 \mu \mathrm{L})$. The experiment was performed at least three times and repeated twice. Endogenous GAPDH was used as a standardized control. Quantitative analysis was performed by comparing CT values.

\section{Flow cytometry analysis}

A single cell suspension from cultured cells or mouse tumors was prepared. The cells were incubated with an $\mathrm{Fc}$ blocking agent and stained with the following fluorescent dye-conjugated antibodies or isotypes for 30 minutes at $4{ }^{\circ} \mathrm{C}$ : mouse anti-CD4, mouse anti-CD25, mouse anti-CD8, mouse antiCD3, small mouse anti-F4/80, mouse anti-CD11b, and mouse anti-NK1.1 (BioLegend). The data was immediately acquired by the FACSAria SORP flow cytometer (BD Biosciences) and analyzed using FlowJo software.

\section{SYTOX green uptake and time-lapse microscopy}

The cells were seeded in a 96-well plate overnight and treated with $10 \mu \mathrm{M}$ Raptinal in the presence of $2.5 \mu \mathrm{M}$ SYTOX green for 2 hours. A microplate reader was used to continuously record the fluorescence at $528 \mathrm{~nm}$ after excitation at $485 \mathrm{~nm}$ every 10 minutes. For time-lapse microscopy, cells seeded in $35 \mathrm{~mm}$ glass bottom dishes overnight were treated with $10 \mu \mathrm{M}$ Raptinal in complete DMEM containing $2.5 \mu \mathrm{M}$ SYTOX green and imaged using a Zeiss 880 laser scanning confocal microscope within an environmental chamber maintained at $37^{\circ} \mathrm{C}$ and $5 \% \mathrm{CO}_{2}$.

\section{LDH detection}

The detection of LDH was performed using the CytoTox $96{ }^{\circledR}$ Non-Radioactive Cytotoxicity Assay Kit (Promega, G1780) according to the manufacturer's recommended protocol.

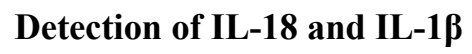

The ELISA kit purchased from Invitrogen (Invitrogen; \#BMS267-2; \# A35574) was used to process the cells according to the protocols provided by the supplier. Then, a microplate reader was used to detect and record the results.

\section{RNA-seq library construction for 10x Genomics single-cell 5' sequencing}

Pancreatic tissues from six mice (three $\mathrm{Kras}^{\mathrm{G} 12 \mathrm{D}}$;PDX1-Cre and three $\mathrm{Kra}^{\mathrm{sG} 12 \mathrm{D}} ; \mathrm{USP} 48^{\text {flox/flox }}$;PDX1- 
1 Cre) were taken and prepared into single cell suspensions. The single cell suspensions were 2 subsequently stained with antibody (CD45-BV421, $0.5 \mathrm{~mL} / 10^{6}$ cells) and then sorted into centrifuge 3 tubes pre-wetted with fetal bovine serum (FBS) using a FACSAria SORP flow cytometer (BD

4 Biosciences). Dead cells were excluded by propidium iodide (PI). A single-cell 5' RNA sequencing 5 library for immunized (CD45+) cells was constructed for each of the six samples separately, 6 according to the protocol of the Chromium Single Cell 5' Library Construction Kit (10x Genomics). 7

\section{Xenograft Tumor Studies}

9 The cells transfected with the corresponding lentivirus or plasmid were made into a cell suspension 10 with a density of $1 \times 10^{7} / \mathrm{mL}$. C57 mice aged 8-12 weeks were selected and $100 \mu \mathrm{L}$ of the cell 11 suspension was subcutaneously injected into the left armpit. Tumor growth was observed according to the experimental needs condition.

\section{Statistical analysis} Statistical analysis was performed using GraphPad Prism 8 software (GraphPad Software, San Diego, CA). The two-tailed Student's t-test was used to determine the significance of the differences between the two independent samples. Pearson's correlation analysis was performed to determine the correlation between the two variables. p-values $<0.05$ were considered statistically significant. All p-values are indicated in the graphs $\left({ }^{*} \mathrm{p}<0.05 ;{ }^{* *} \mathrm{p}<0.01 ; * * \mathrm{p}<0.001 ; * * * \mathrm{p}<0.0001 ;\right.$ n.s., not significant). 
1

2

31

4

52

6

7

83

9

104

11

12

13

14

15

16

17

18

19

20

21

22

23

24

25

26

27

28

29

30

31

32

33

34

35

36

37

Kovacs, S. B. \& Miao, E. A. Gasdermins: Effectors of Pyroptosis. Trends in cell biology 27, 673-684, doi:10.1016/j.tcb.2017.05.005 (2017).

2 Zhou, Z. et al. Granzyme A from cytotoxic lymphocytes cleaves GSDMB to trigger pyroptosis in target cells. Science (New York, N.Y.) 368, doi:10.1126/science.aaz7548 (2020).

Humphries, F. et al. Succination inactivates gasdermin D and blocks pyroptosis. Science (New York, N.Y.) 369, 1633-1637, doi:10.1126/science.abb9818 (2020).

Ding, J. et al. Pore-forming activity and structural autoinhibition of the gasdermin family. Nature 535, 111-116, doi:10.1038/nature18590 (2016).

5 Wang, Q. et al. A bioorthogonal system reveals antitumour immune function of pyroptosis. Nature 579, 421-426, doi:10.1038/s41586-020-2079-1 (2020).

6 Xia, X. et al. The role of pyroptosis in cancer: pro-cancer or pro-"host"? Cell death \& disease 10, 650, doi:10.1038/s41419-019-1883-8 (2019).

7 Wang, Y. et al. Chemotherapy drugs induce pyroptosis through caspase- 3 cleavage of a gasdermin. Nature 547, 99-103, doi:10.1038/nature22393 (2017).

8 De Schutter, E. et al. GSDME and its role in cancer: From behind the scenes to the front of the stage. International journal of cancer 148, 2872-2883, doi:10.1002/ijc.33390 (2021).

9 Ibrahim, J., De Schutter, E. \& Op de Beeck, K. GSDME: A Potential Ally in Cancer Detection and Treatment. Trends in cancer 7, 392-394, doi:10.1016/j.trecan.2020.12.002 (2021).

10 Tan, G., Huang, C., Chen, J. \& Zhi, F. HMGB1 released from GSDME-mediated pyroptotic epithelial cells participates in the tumorigenesis of colitis-associated colorectal cancer through the ERK1/2 pathway. Journal of hematology \& oncology 13, 149, doi:10.1186/s13045-020-00985-0 (2020).

11 Zhang, X. et al. Miltirone induces cell death in hepatocellular carcinoma cell through GSDME-dependent pyroptosis. Acta pharmaceutica Sinica. B 10, 1397-1413, doi:10.1016/j.apsb.2020.06.015 (2020).

12 Lu, Y., Hou, K., Li, M., Wu, X. \& Yuan, S. Exosome-Delivered LncHEIH Promotes Gastric Cancer Progression by Upregulating EZH2 and Stimulating Methylation of the GSDME Promoter. Frontiers in cell and developmental biology 8, 571297, doi:10.3389/fcell.2020.571297 (2020).

13 Cai, J. et al. Natural product triptolide induces GSDME-mediated pyroptosis in head and neck cancer through suppressing mitochondrial hexokinase-II. Journal of experimental \& clinical cancer research : CR 40, 190, doi:10.1186/s13046-021-01995-7 (2021).

14 Zhang, Z. et al. Gasdermin E suppresses tumour growth by activating anti-tumour immunity. Nature 579, 415-420, doi:10.1038/s41586-020-2071-9 (2020).

5 Liu, Y. et al. Gasdermin E-mediated target cell pyroptosis by CAR T cells triggers cytokine release syndrome. Science immunology 5, doi:10.1126/sciimmunol.aax7969 (2020).

16 Dikic, I., Wakatsuki, S. \& Walters, K. J. Ubiquitin-binding domains - from structures to functions. Nature reviews. Molecular cell biology 10, 659-671, doi:10.1038/nrm2767 (2009).

1 Ikeda, F. \& Dikic, I. Atypical ubiquitin chains: new molecular signals. 'Protein Modifications: Beyond the Usual Suspects' review series. EMBO reports 9, 536-542, 
doi:10.1038/embor.2008.93 (2008).

Ernst, A. et al. A strategy for modulation of enzymes in the ubiquitin system. Science (New York, N.Y.) 339, 590-595, doi:10.1126/science.1230161 (2013).

19 Harris, I. S. et al. Deubiquitinases Maintain Protein Homeostasis and Survival of Cancer Cells upon Glutathione Depletion. Cell metabolism 29, 1166-1181.e1166, doi:10.1016/j.cmet.2019.01.020 (2019).

20 Gutierrez-Diaz, B. T., Gu, W. \& Ntziachristos, P. Deubiquitinases: Pro-oncogenic Activity and Therapeutic Targeting in Blood Malignancies. Trends in immunology 41, 327-340, doi:10.1016/j.it.2020.02.004 (2020).

21 Ashkenazi, A. et al. Polyglutamine tracts regulate beclin 1-dependent autophagy. Nature 545, 108-111, doi:10.1038/nature22078 (2017).

22 Sharma, A. et al. USP14 regulates DNA damage repair by targeting RNF168-dependent ubiquitination. Autophagy 14, 1976-1990, doi:10.1080/15548627.2018.1496877 (2018).

23 Lork, M., Verhelst, K. \& Beyaert, R. CYLD, A20 and OTULIN deubiquitinases in NF-KB signaling and cell death: so similar, yet so different. Cell death and differentiation 24 , 1172-1183, doi:10.1038/cdd.2017.46 (2017).

24 Zhou, Y. et al. High-throughput screening of a CRISPR/Cas9 library for functional genomics in human cells. Nature 509, 487-491, doi:10.1038/nature13166 (2014).

25 Shi, J. et al. Cleavage of GSDMD by inflammatory caspases determines pyroptotic cell death. Nature 526, 660-665, doi:10.1038/nature15514 (2015).

26 Harrigan, J. A., Jacq, X., Martin, N. M. \& Jackson, S. P. Deubiquitylating enzymes and drug discovery: emerging opportunities. Nature reviews. Drug discovery 17, 57-78, doi:10.1038/nrd.2017.152 (2018).

27 Zhou, A. et al. Gli1-induced deubiquitinase USP48 aids glioblastoma tumorigenesis by stabilizing Gli1. EMBO reports 18, 1318-1330, doi:10.15252/embr.201643124 (2017).

$28 \mathrm{Li}$, S. et al. The deubiquitinating enzyme USP48 stabilizes TRAF2 and reduces E-cadherinmediated adherens junctions. FASEB journal : official publication of the Federation of American Societies for Experimental Biology 32, 230-242, doi:10.1096/fj.201700415RR (2018).

29 Cetkovská, K., šustová, H. \& Uldrijan, S. Ubiquitin-specific peptidase 48 regulates Mdm2 protein levels independent of its deubiquitinase activity. Scientific reports 7, 43180, doi:10.1038/srep43180 (2017).

30 Uckelmann, M. et al. USP48 restrains resection by site-specific cleavage of the BRCA1 ubiquitin mark from H2A. Nature communications 9, 229, doi:10.1038/s41467-01702653-3 (2018).

31 Nakamura, N. Ubiquitin System. International journal of molecular sciences 19, doi:10.3390/ijms19041080 (2018).

32 Du, L. et al. USP48 Is Upregulated by Mettl14 to Attenuate Hepatocellular Carcinoma via Regulating SIRT6 Stabilization. Cancer research 81, 3822-3834, doi:10.1158/00085472.Can-20-4163 (2021).

33 Tang, R. et al. Ferroptosis, necroptosis, and pyroptosis in anticancer immunity. Journal of hematology \& oncology 13, 110, doi:10.1186/s13045-020-00946-7 (2020).

34 Baxi, S. et al. Immune-related adverse events for anti-PD-1 and anti-PD-L1 drugs: systematic review and meta-analysis. BMJ (Clinical research ed.) $\mathbf{3 6 0}, \mathbf{k 7 9 3 ,}$ 
doi:10.1136/bmj.k793 (2018).

35 Mevissen, T. E. T. \& Komander, D. Mechanisms of Deubiquitinase Specificity and Regulation. Annual review of biochemistry 86, 159-192, doi:10.1146/annurev-biochem061516-044916 (2017).

6 Shi, J., Gao, W. \& Shao, F. Pyroptosis: Gasdermin-Mediated Programmed Necrotic Cell

37 Cookson, B. T. \& Brennan, M. A. Pro-inflammatory programmed cell death. Trends in microbiology 9, 113-114, doi:10.1016/s0966-842x(00)01936-3 (2001).

38 Zhao, P. et al. Programming cell pyroptosis with biomimetic nanoparticles for solid tumor immunotherapy. Biomaterials 254, 120142, doi:10.1016/j.biomaterials.2020.120142 (2020).

39 Erkes, D. A. et al. Mutant BRAF and MEK Inhibitors Regulate the Tumor Immune Microenvironment via Pyroptosis. Cancer discovery 10, 254-269, doi:10.1158/21598290.Cd-19-0672 (2020).

$40 \mathrm{Hou}$, J., Hsu, J. M. \& Hung, M. C. Molecular mechanisms and functions of pyroptosis in inflammation and antitumor immunity. Molecular cell, doi:10.1016/j.molcel.2021.09.003 (2021).

41 Hugo, W. et al. Genomic and Transcriptomic Features of Response to Anti-PD-1 Therapy in Metastatic Melanoma. Cel/ 165, 35-44, doi:10.1016/j.cell.2016.02.065 (2016).

42 Cortellini, A. et al. Integrated analysis of concomitant medications and oncological outcomes from PD-1/PD-L1 checkpoint inhibitors in clinical practice. Journal for immunotherapy of cancer 8, doi:10.1136/jitc-2020-001361 (2020).

Funding: This research was supported by grant from National Natural Science Foundation of China (82172350, 81874040 and 82002228), Shandong Key Research and Development Program (2019GSF108218), Taishan Scholars Climbing Program of Shandong Province (tspd20210323), Young Taishan Scholars Program of Shandong Province (tsqn201909176 and tsqn201909177), Natural Science Foundation of Shandong Province (ZR2020QH280), Qilu Young Scholars Program of Shandon University. 
Fig 1

A

CRISPR knockout
pooled sgRNA for

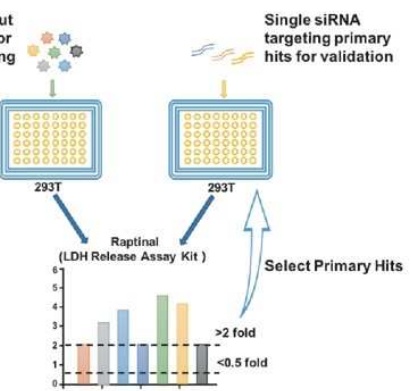

B

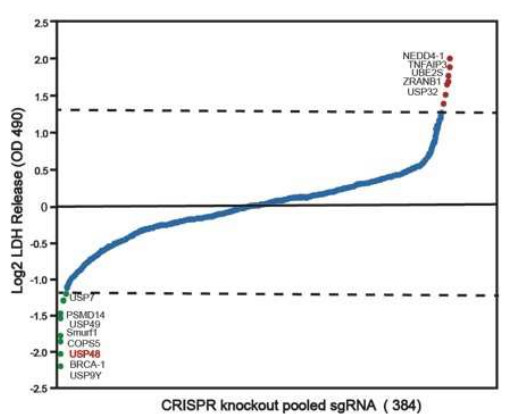

C

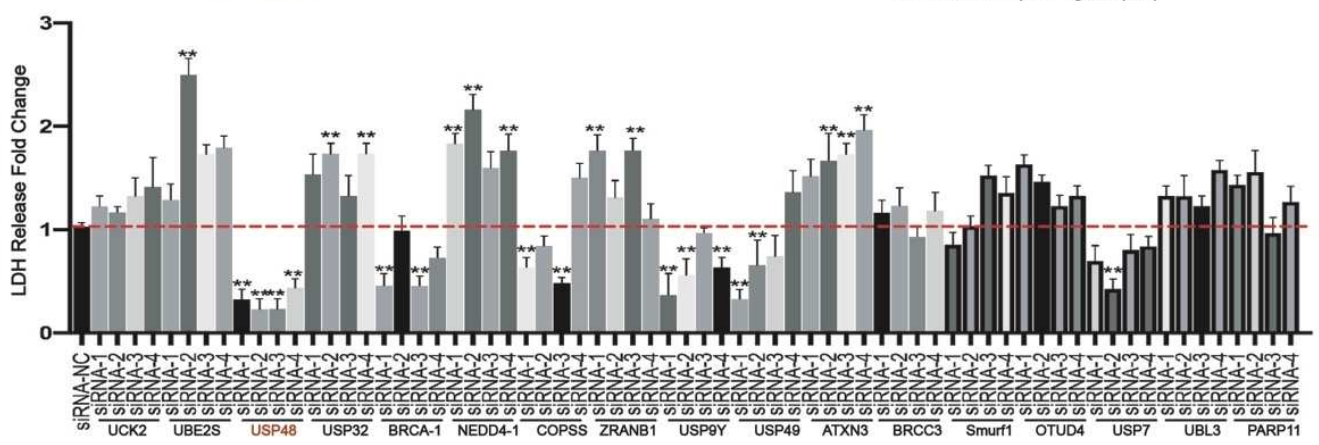

D

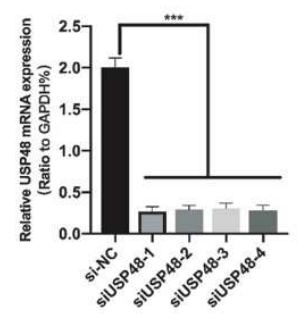

$\mathbf{F}$

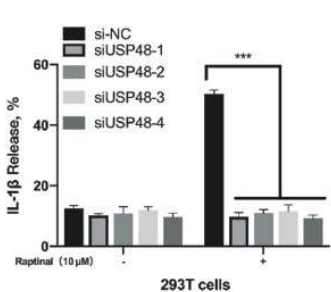

I

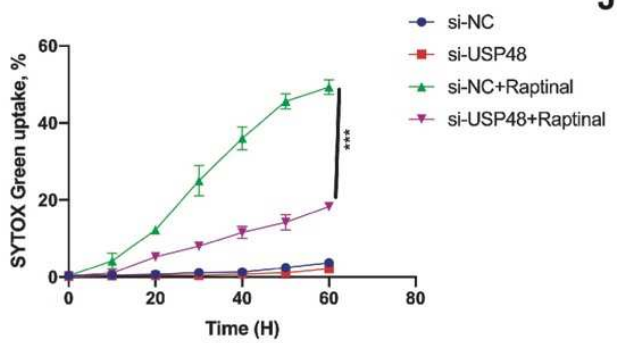

H

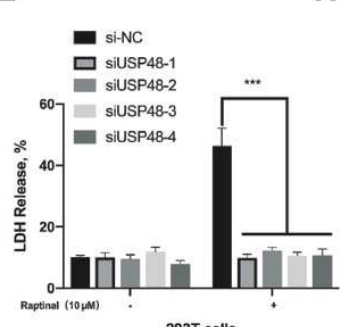

G

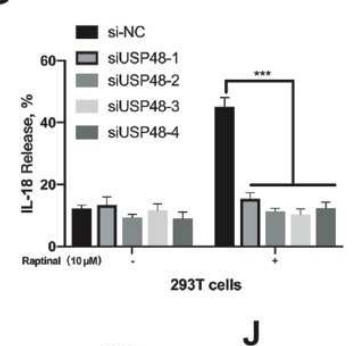

Raptinal
$(10 \mu \mathrm{M})$ $(10 \mu \mathrm{M}) \quad 10$

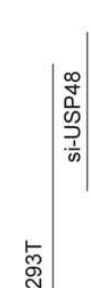

$$
\text { Raptinal }
$$$$
\text { 今े HMGB1 }
$$$$
\text { HMGB1 - - - - - - - }
$$

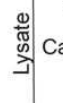

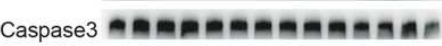

293T

3 Figure 1. USP48 is involved in the regulation of pyroptosis.

4 (A) Two-stage screening strategy diagram of the CRISPR Knockout Pooled Lentiviral sgRNA

5 Library and siRNA verification. (B) The fold change of LDH secretion in 293T cells transfected with 1114 targeted sgRNAs after being treated with $10 \mu \mathrm{M}$ Raptinal for 1 hour; the targeted genes that significantly increase or decrease LDH secretion are highlighted in red or green, respectively. 
1 (C) Fold changes of LDH secretion in $293 \mathrm{~T}$ cells transfected with siRNAs for secondary validation

2 followed by treatment with Raptinal as in (B). Data are normalized to untreated 293T cells (dashed

3 line). (D) qRT-PCR verifies the transfection efficiency of si-NC and USP48-specific siRNA in 293T

4 cells. (E) The LDH detection kit was used to analyze the changes in LDH levels in 293T cells

5 transfected with siRNA (si-NC) or siRNA specific to USP48 (si-USP48) after treatment with

6 Raptinal. (F) An ELISA experiment was used to analyze the expression changes of IL-1 $\beta$ in 293T

7 cells transfected with siRNA (si-NC) or siRNA specific to USP48 (si-USP48) after treatment with

8 Raptinal. (G) An ELISA experiment was used to analyze the expression changes of IL-18 in 293T

9 cells transfected with siRNA (si-NC) or siRNA specific to USP48 (si-USP48) after treatment with

10 Raptinal. (H) Time-lapse microscopy observed the morphological changes and the absorption of

11 SYTOX green of 293T cells transfected with siRNA (si-NC) or USP48 specific siRNA (si-USP48) after treatment with Raptinal. (I) Quantitative analysis chart of SYTOX green absorption. (J) Kinetics of caspase-3 and GSDME cleavage and HMGB1 release by immunoblotting of cell lysates

14 and culture supernatants. Data are shown as mean \pm SD of three independent experiments. ${ }^{*} \mathrm{p}<0.05$; ${ }^{* *} \mathrm{p}<0.01 ; * * * \mathrm{p}<0.001 ;{ }^{* * * *} \mathrm{p}<0.0001 ;$ n.s., not significant; scale bar, $20 \mu \mathrm{m}$. 
Fig 2

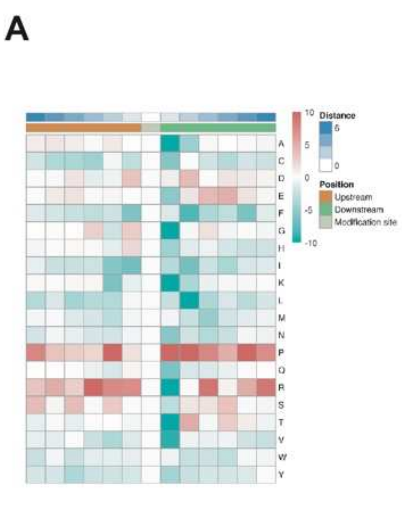

B

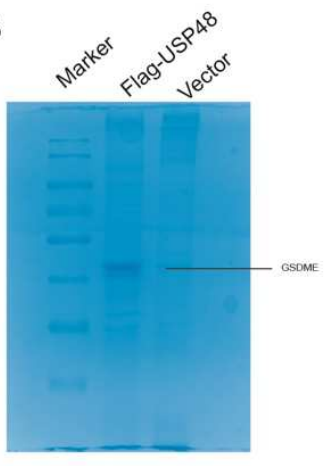

C

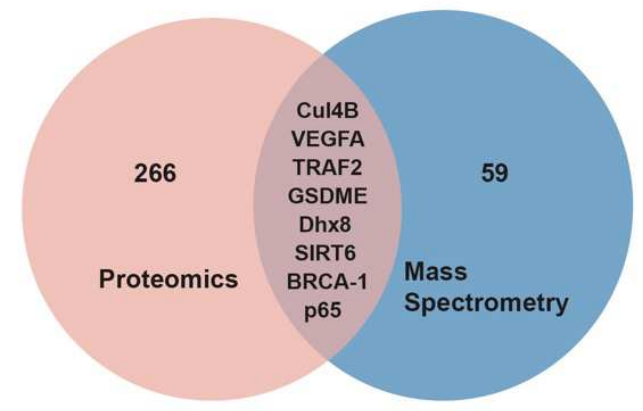

D

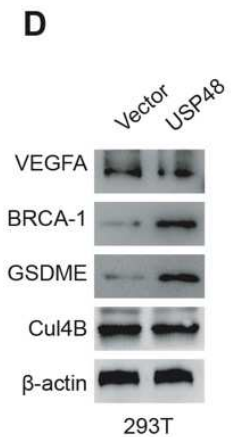

E

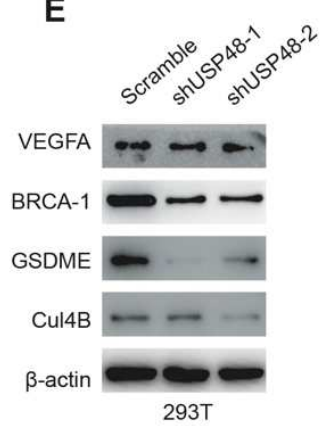

$\mathrm{F}$

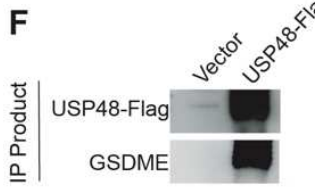

\begin{tabular}{r|r} 
USP48-Flag $=$ \\
GSDME \\
B-actin
\end{tabular}

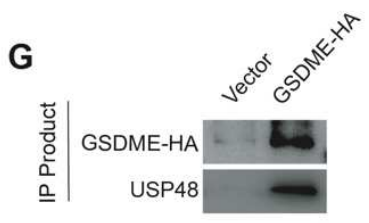

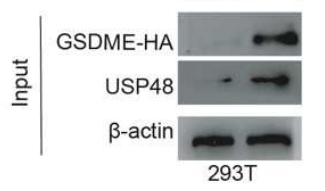

H

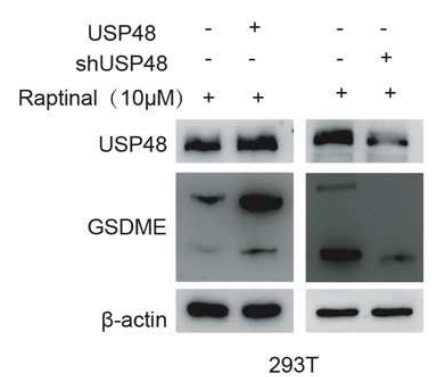

1

2 Figure 2. GSDME is a downstream target protein of USP48.

3 (A) The heat map summarizes the difference in protein expression in $293 \mathrm{~T}$ cells with normal

4 expression of USP48 and overexpression of USP48. (B) The Flag-USP48 pull-down product from

$5293 \mathrm{~T}$ cells was separated by SDS-PAGE, visualized by silver staining, and the protein interacting

6 with USP48 was identified by mass spectrometry. (C) Joint analysis of proteomics results and mass

7 spectrometry sequencing results to get an intersection. (D and E) Verification of the results obtained

8 in (C) by Western blotting. (F) Lysates from cells expressing the control or the indicated constructs

9 were immunoprecipitated (IP) with IgG or anti-USP48 and then immunoblotted with anti-Flag. (G)

10 Lysates from cells expressing the control or the indicated constructs were immunoprecipitated (IP)

11 with IgG or anti-GSDME and then immunoblotted with anti-HA. (H) Western blotting of USP48

12 and GSDME in Raptinal-treated 293T cells transfected with different combinations of control vector,

13 USP48 vector and/or sh-GSDME. 
Fig 3

A

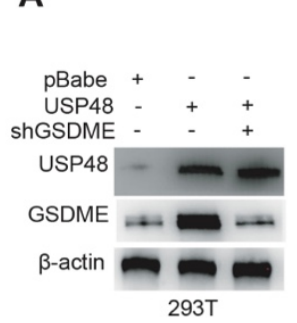

D

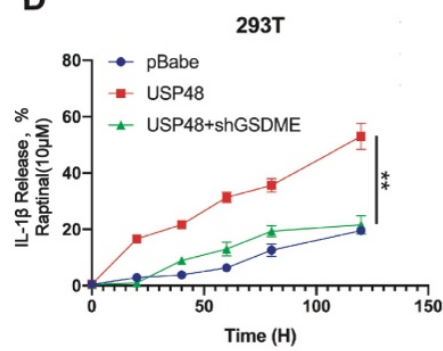

G

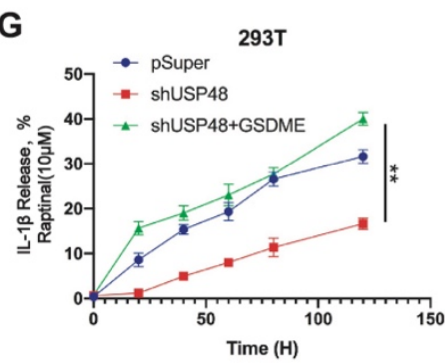

B

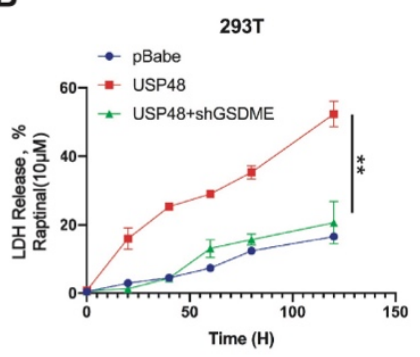

C

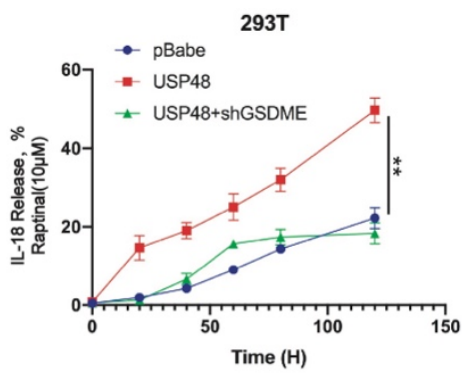

E

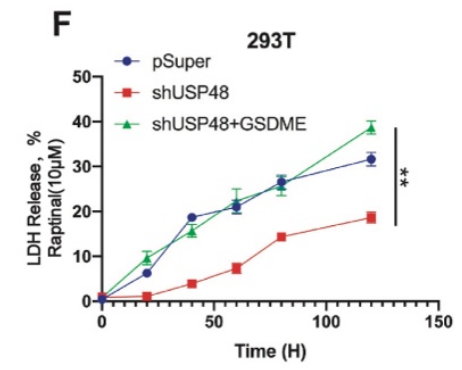

$\mathbf{J}$

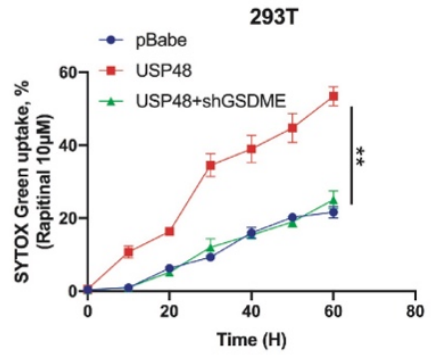

I
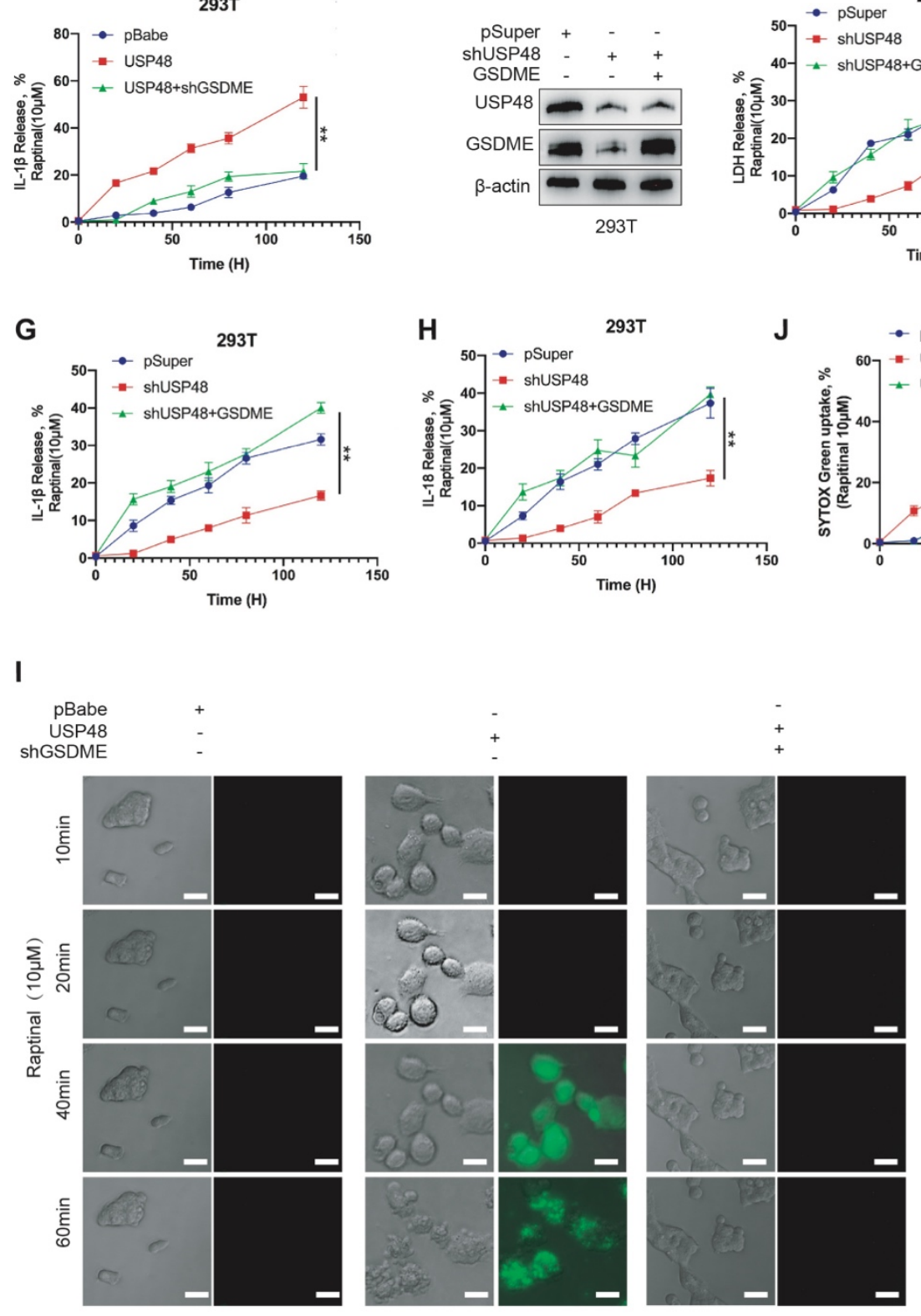

2 Figure 3. USP48 regulates pyroptosis through GSDME.

3 (A) Western blotting of USP48 and GSDME in 293T cells transfected with different combinations

4 of control vector, USP48 vector, and/or sh-GSDME. (B) Measurement of LDH production in

5 transformed cells described in (A). (C) Measurement of IL-18 production in transformed cells 
1 described in (A). (D) Measurement of IL-1 $\beta$ production in transformed cells described in (A). (E)

2 Western blotting of USP48 and GSDME in 293T cells transfected with different combinations of 3 control vector, shUSP48 vector, and/or GSDME. (F) Measurement of LDH production in 4 transformed cells described in (F). (G) Measurement of IL-1 $\beta$ production in transformed cells 5 described in (F). (H) Measurement of IL-18 production in transformed cells described in (F). (I-J)

6 Time-lapse microscopy observed the morphological changes and the absorption of SYTOX green 7 in 293T cells transfected with different combinations of control vector, USP48 vector, and/or sh-

8 GSDME. Data are shown as mean \pm SD of three independent experiments. ${ }^{*} \mathrm{p}<0.05 ;{ }^{* *} \mathrm{p}<0.01$; $9 \quad * * * \mathrm{p}<0.001 ; * * * * \mathrm{p}<0.0001 ;$ n.s., not significant; scale bar, $20 \mu \mathrm{m}$. 
Fig 4
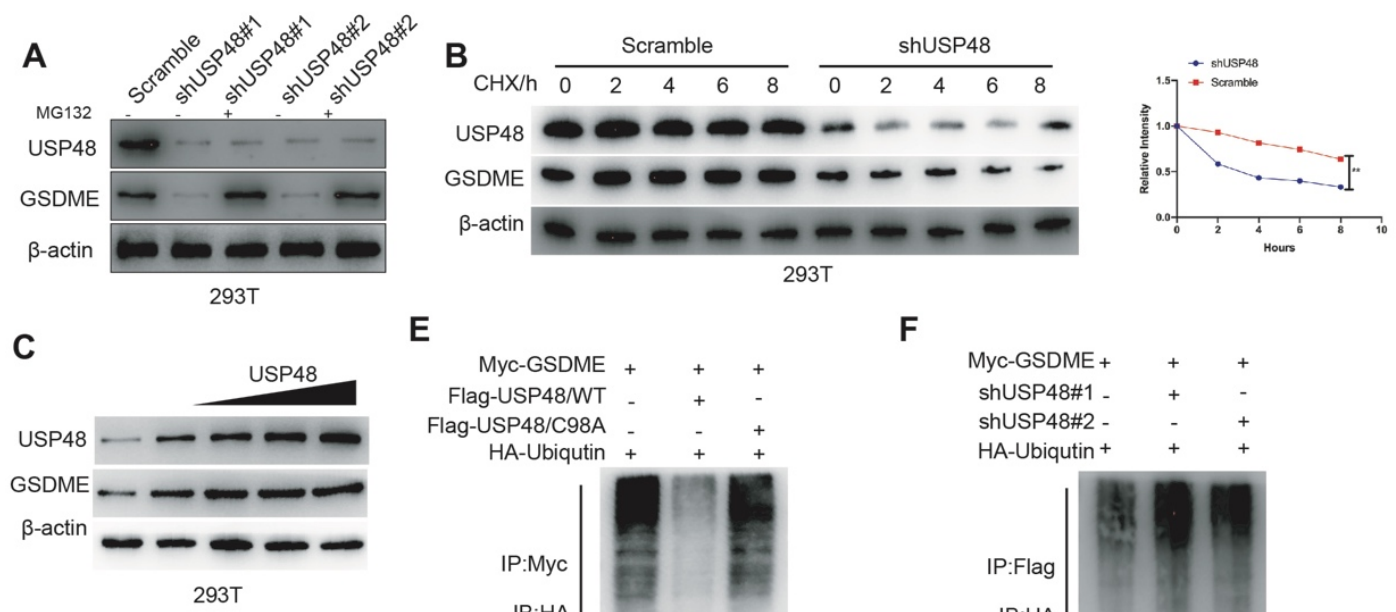

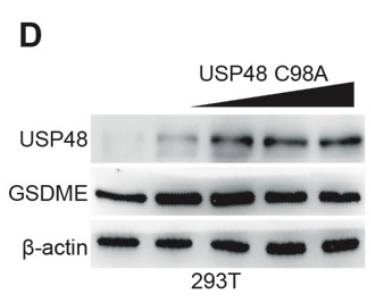

E

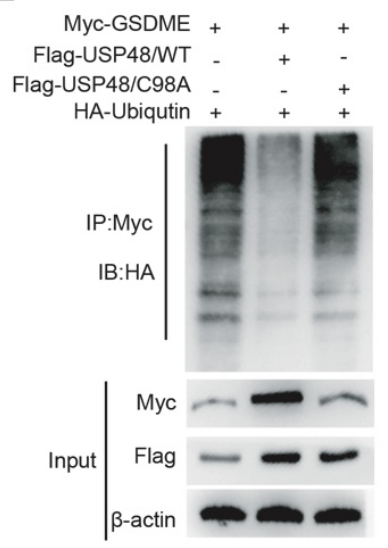

H
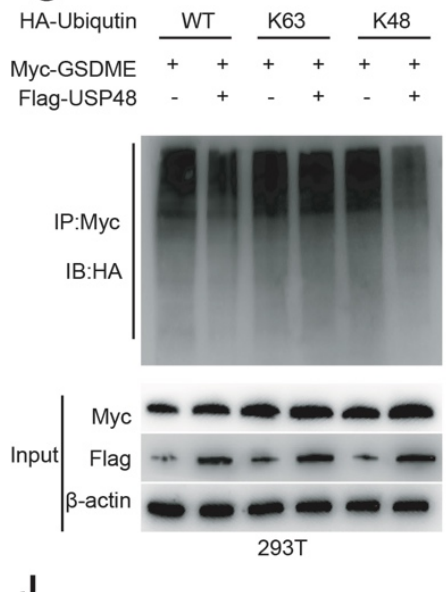

K30 SNLNDSD K QLLSLV

K3OR SNLNDSD R QLLSLV

K120 SSFGTLR KEVDLQQ

K120R SSFGTLR REVDLQQ

K189 GIVGIQT K VQVSAT

K189R GIVGIQT R VQVSAT
F

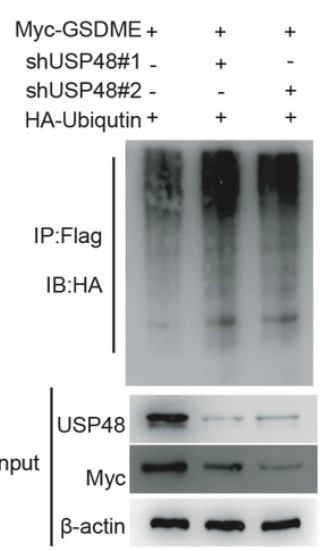

|

Myc-GSDME 々々官 HA-Ubiquitin(K48) -++++++++ $\begin{array}{lccccccc} & 30 & 39 & 120 & 161 & 189 & 240 & 248 \\ \text { WT } & \mathrm{K} & \mathrm{K} & \mathrm{K} & \mathrm{K} & \mathrm{K} & \mathrm{K} & \mathrm{K} \\ \mathrm{K} 30 & \mathrm{~K} & \mathrm{R} & \mathrm{R} & \mathrm{R} & \mathrm{R} & \mathrm{R} & \mathrm{R} \\ \mathrm{K} 39 & \mathrm{R} & \mathrm{K} & \mathrm{R} & \mathrm{R} & \mathrm{R} & \mathrm{R} & \mathrm{R} \\ \mathrm{K} 120 \mathrm{R} & \mathrm{R} & \mathrm{K} & \mathrm{R} & \mathrm{R} & \mathrm{R} & \mathrm{R} \\ \mathrm{K} 161 \mathrm{R} & \mathrm{R} & \mathrm{R} & \mathrm{K} & \mathrm{R} & \mathrm{R} & \mathrm{R} \\ \mathrm{K} 189 \mathrm{R} & \mathrm{R} & \mathrm{R} & \mathrm{R} & \mathrm{K} & \mathrm{R} & \mathrm{R} \\ \mathrm{K} 240 \mathrm{R} & \mathrm{R} & \mathrm{R} & \mathrm{R} & \mathrm{R} & \mathrm{K} & \mathrm{R} \\ \mathrm{K} 248 \mathrm{R} & \mathrm{R} & \mathrm{R} & \mathrm{R} & \mathrm{R} & \mathrm{R} & \mathrm{K}\end{array}$

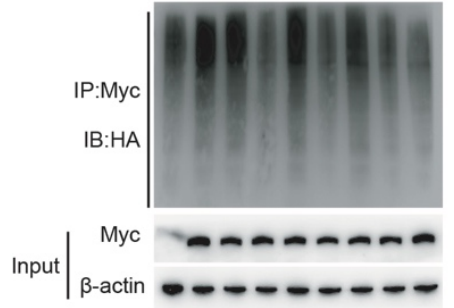

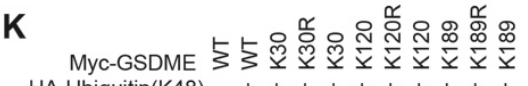
HA-Ubiquitin(K48) - + + + + + + + + Flag-USP48 - . . . + . + .
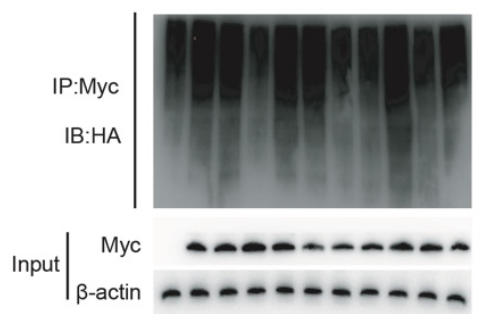

2 Figure 4. USP48 inhibits the degradation of GSDME by deubiquitinating it.

3 (A) In the absence or presence of the proteasome inhibitor MG132 $(50 \mu \mathrm{g} / \mathrm{mL})$, the levels of USP48 and GSDME proteins in cells transfected with control or USP48 shRNA were detected by Western blotting. (B) Within the specified hours, in the absence or presence of the protein synthesis inhibitor cycloheximide (CHX; $50 \mu \mathrm{g} / \mathrm{mL}$ ), Western blotting was used to detect USP48 and GSDME in 293T cells transfected with control or USP48 shRNA protein levels. (C and D) Western blotting to 
1 measure USP48 and GSDME protein levels in cells with Dox-inducible expression of wild-type 2 USP48 (USP48/WT) (C) and a catalytically inactive mutant of USP48 (USP48/C98A) (D). (E) 3 Lysates from cells expressing GSDME, USP48, and HA-Ub were pulled down with anti-Myc and 4 then immunoblotted with anti-HA (top). Input is immunoblotting with anti-Myc and anti-Flag 5 (bottom). $\beta$-actin is used as a loading control. (F) Lysates from cells expressing GSDME, shUSP48, 6 and HA-Ub were pulled down with anti-Flag and then immunoblotted with anti-HA (top). Input is 7 immunoblotting with anti-USP48 and anti-Flag (bottom). $\beta$-actin is used as a loading control. (G) 8 Lysates from cells expressing GSDME, USP48, and ubiquitin mutants were pulled down with anti9 Myc and then immunoblotted with anti-HA (top). Input is immunoblotting with anti-Myc and anti10 Flag (bottom). $\beta$-actin is used as a loading control. (H) A series of GSDME ubiquitination site mutants. (I) The cell lysate expressing the designated GSDME ubiquitination site mutant was pulled down with anti-Myc and then immunoblotted with anti-HA (top). Enter anti-Myc and anti-Flag (bottom) for Western blotting. $\beta$-actin was used as a loading control. (J) The lysine site associated with GSDME was mutated to arginine. (K) Using anti-Myc to pull down the lysate of cells expressing the specified GSDME mutant and then using anti-HA for immunoblotting (top). Input is immunoblotting with anti-Myc and anti-Flag (bottom). $\beta$-actin was used as a loading control. Data are shown as mean $\pm \mathrm{SD}$ of three independent experiments. $* * \mathrm{p}<0.01$. 
Fig5

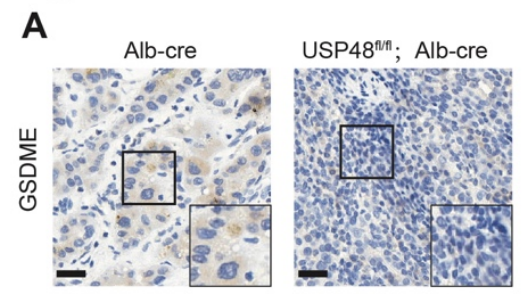

DEN

D

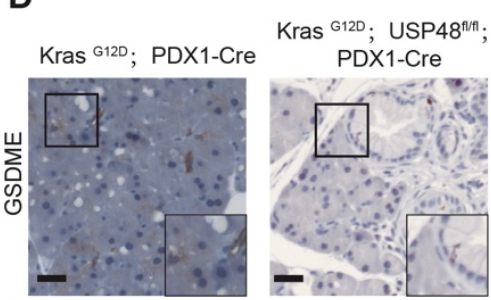

G

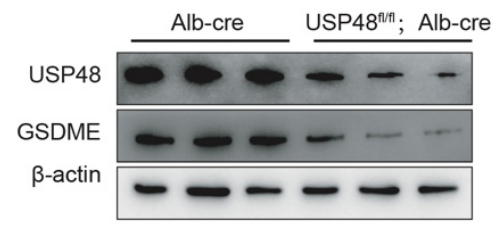

I
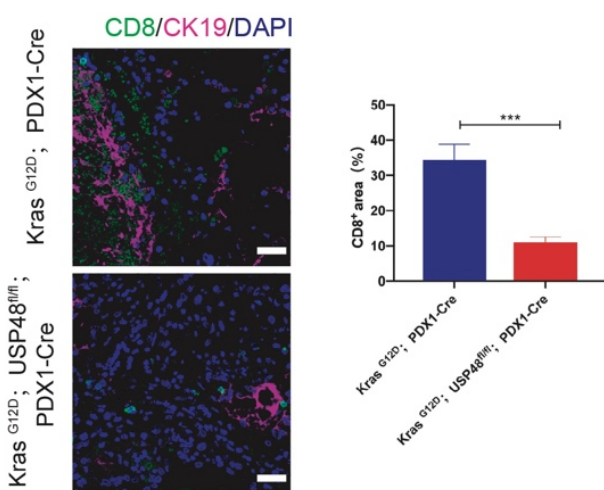

H

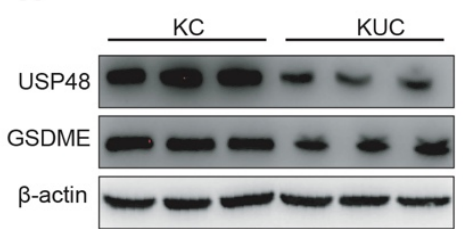

J

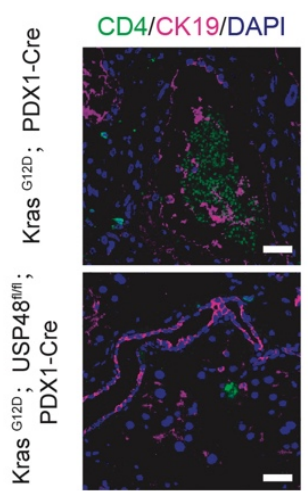

C

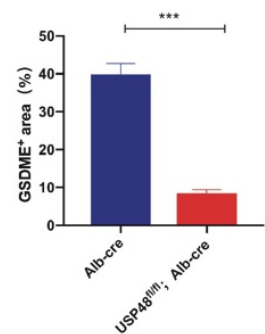

$\mathbf{F}$

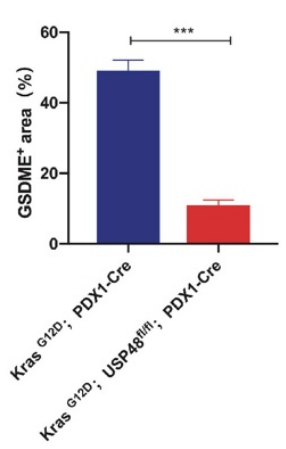

$\mathbf{K}$

K Kras ${ }^{6120}$; PDX1-Cre ${ }_{\text {Kras }}^{\text {G120; USP48 }}$ PDX1-Cre
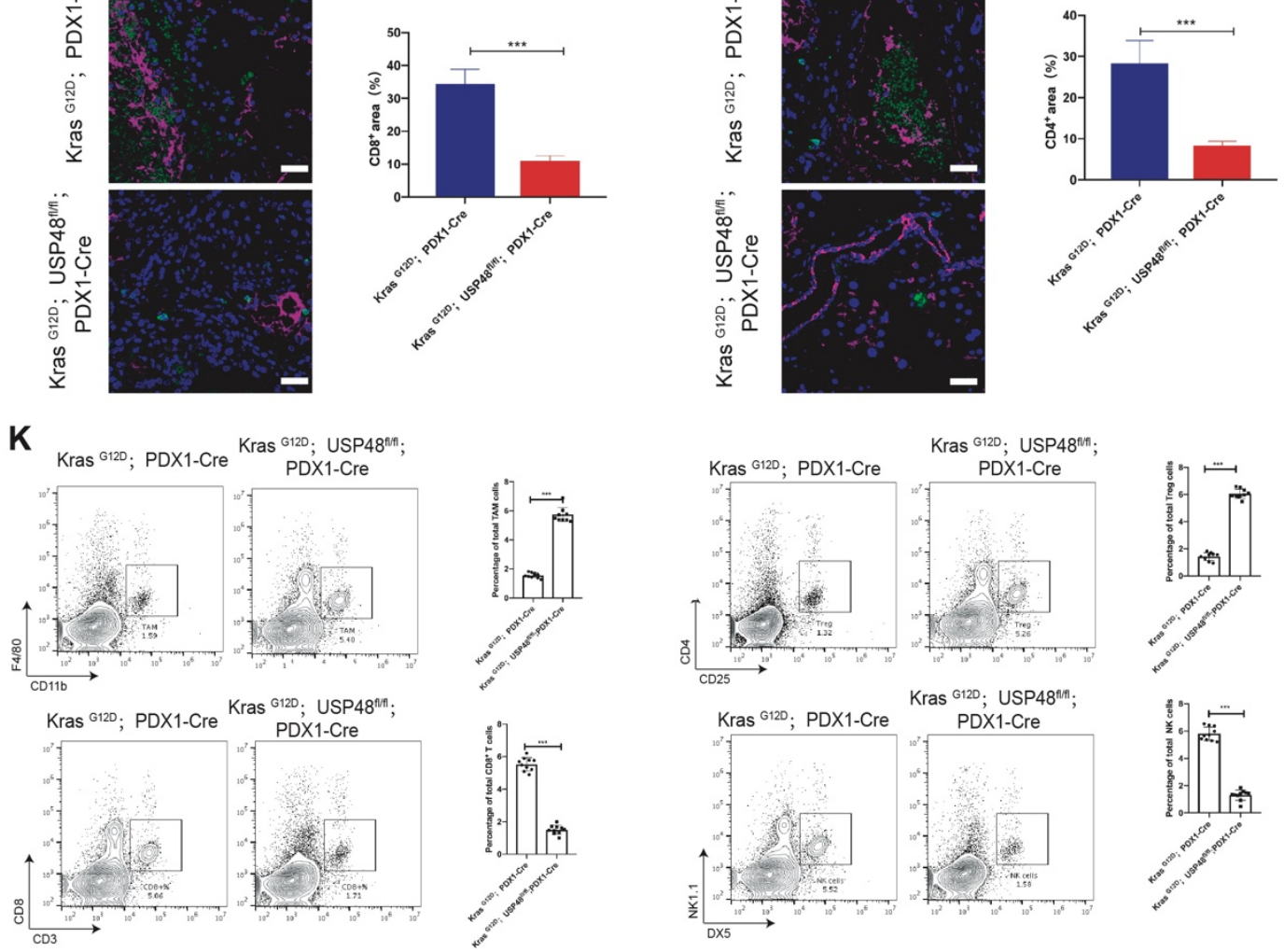

2 Figure 5. USP48 is involved in regulating anti-tumor immunity.

3 (A) Immunohistochemistry was used to detect the expression of USP48 and GSDME in liver cancer

4 tissues of Alb-Cre;USP48 $8^{\text {flox fllox }}$ and Alb-Cre;USP48 ${ }^{\text {WT }}$ mice. (B) Immunofluorescence was used to

5 detect the expression of USP48 and GSDME in liver cancer tissues of Alb-Cre;USP48 $8^{\text {flox flox }}$ and 
1 Alb-Cre;USP48 ${ }^{\mathrm{WT}}$ mice. (C) Quantification of (B). (D) Immunohistochemistry was used to detect

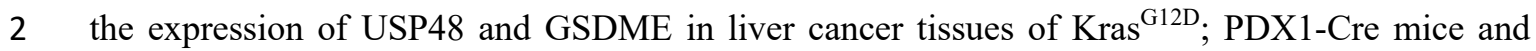
$3 \mathrm{Kras}^{\mathrm{G} 12 \mathrm{D}}$;USP48 $8^{\mathrm{fl} / \mathrm{fl}}$;PDX1-Cre mice. (E) Immunofluorescence was used to detect the expression of 4 USP48 and GSDME in liver cancer tissues of $\mathrm{Kras}^{\mathrm{G} 12 \mathrm{D}}$;PDX1-Cre mice and $5 \quad \mathrm{Kras}^{\mathrm{G} 12 \mathrm{D}}$;USP48 $8^{\mathrm{fl} / \mathrm{fl}}$;PDX1-Cre mice. (F) Quantification of (E). (G) Western blotting was used to 6 detect the expression of USP48 and GSDME in liver cancer tissues of Alb-Cre;USP48 $8^{\text {flox fllox }}$ mice 7 and Alb-Cre;USP48 ${ }^{\mathrm{WT}}$ mice. (H) Western blotting was used to detect the expression of USP48 and 8 GSDME in liver cancer tissues of $\mathrm{Kras}^{\mathrm{G} 12 \mathrm{D}}$;PDX1-Cre mice and $\mathrm{Kras}^{\mathrm{G} 12 \mathrm{D}}$;USP48 $8^{\mathrm{fl} / \mathrm{fl}}$;PDX1-Cre 9 mice. (I) Multicolor immunofluorescence to detect the distribution of CD8+ T cells in the PDAC 10 tissues of mice. (J) Multicolor immunofluorescence to detect the distribution of CD4+ T cells in the 11 PDAC tissues of mice. (K) Flow cytometry assay to detect the proportion of NK cells, CD8+ T cells, 12 TAMs and Tregs in the pancreatic cancer tissues of $\mathrm{Kras}^{\mathrm{G} 12 \mathrm{D}}$;PDX1-Cre mice and $13 \mathrm{Kras}^{\mathrm{G} 12 \mathrm{D}} ; \mathrm{USP} 48^{\mathrm{fl} / \mathrm{fl}} ; \mathrm{PDX} 1-\mathrm{Cre}$ mice. Data are shown as mean $\pm \mathrm{SD}$ of three independent 14 experiments. ${ }^{*} \mathrm{p}<0.05 ;{ }^{* *} \mathrm{p}<0.01 ;{ }^{* *} \mathrm{p}<0.001 ; * * * \mathrm{p}<0.0001 ;$ n.s., not significant; scale bar, $15 \quad 20 \mu \mathrm{m}$. 


\section{Fig 6}

A
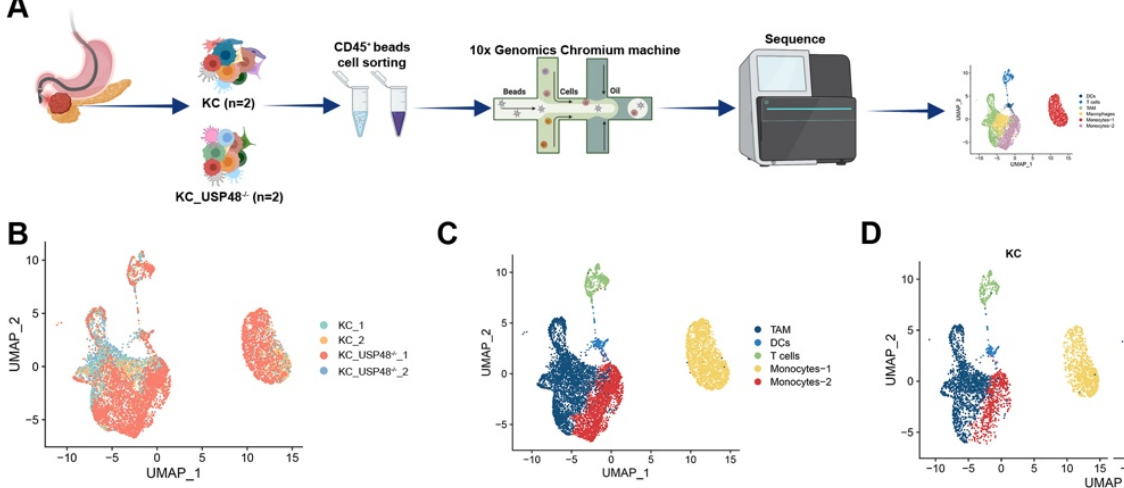

C
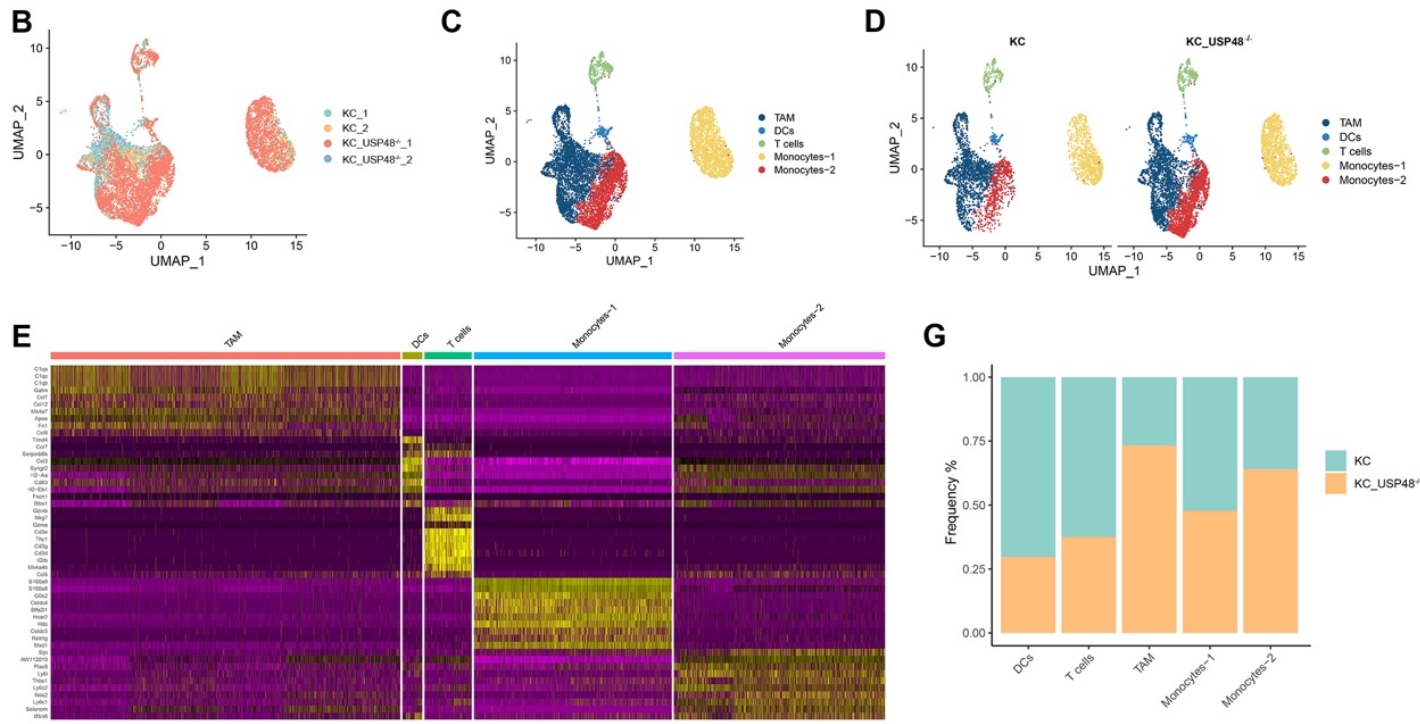

G
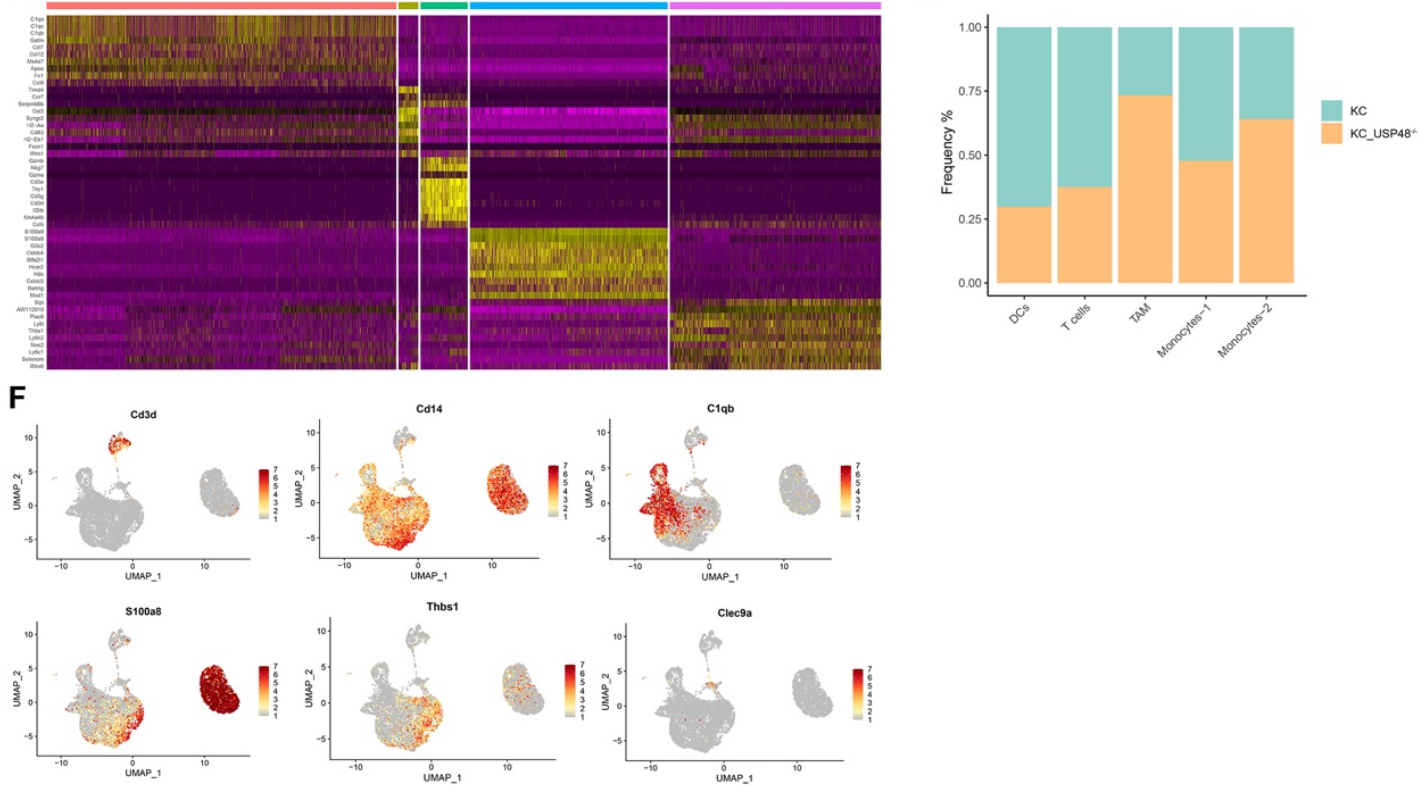

2 Figure 6. Single cell analysis of $K C$ and $K C$;USP48-/- CD45+ cells in pancreatic cancer.

3 (A) KC $(\mathrm{n}=2)$ and $\mathrm{KC}$;USP48-/- $(\mathrm{n}=2)$ pancreatic cancer tissues were collected for digestion to

4 obtain single cells, CD45-labeled magnetic beads were used for analysis, and sequenced after 10x

5 Genomics library construction; (B) UMAP displayed $\mathrm{KC}(\mathrm{n}=2)$ and $\mathrm{KC}$; cell distribution in USP48-

6 /- $(\mathrm{n}=2)$. (C) Group annotation of the obtained single cells (TAMs: tumor-associated macrophages;

7 DCs: dendritic cells). (D) Comparison of $\mathrm{KC}(\mathrm{n}=2)$ and $\mathrm{KC} ; \mathrm{USP} 48-/-(\mathrm{n}=2)$ differences in cell

8 subgroups in pancreatic cancer tissues (it can be clearly seen that there are obvious differences in the number of TAMs and monocyte-2 cells). (E) Marker genes in each cell subgroup (as a basis for grouping). (F) Dot diagram showing each subgroup marker gene ( $\mathrm{Cd} 3 \mathrm{~d}=\mathrm{T}$ cells; $\mathrm{Cd} 14=$ monocytes; $\mathrm{C} 1 \mathrm{qb}=$ ATMs; S100a3 and Thbs1 = monocytes; and Clec9a = DCs). (G) The proportion of each subgroup of cells in $\mathrm{KC}(\mathrm{n}=2)$ and $\mathrm{KC} ; \mathrm{USP}_{4} 8^{-/-}(\mathrm{n}=2)$ pancreatic cancer tissues. 


\section{Fig 7}

A

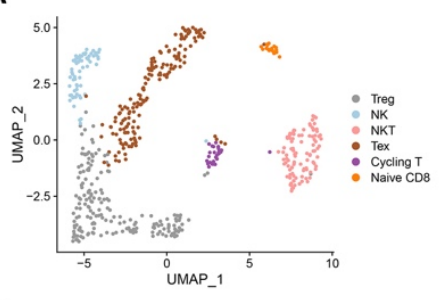

c
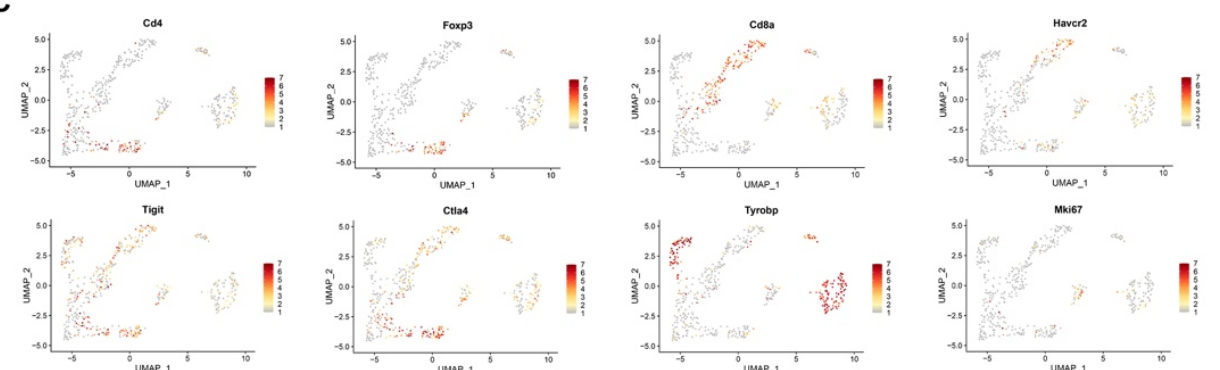

D

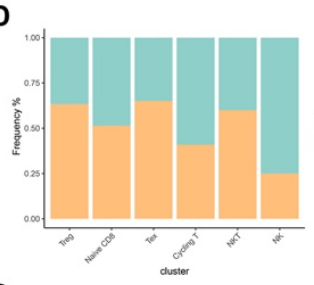

G
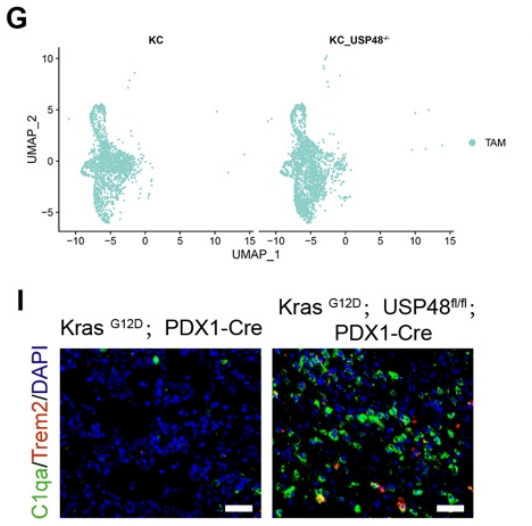

B
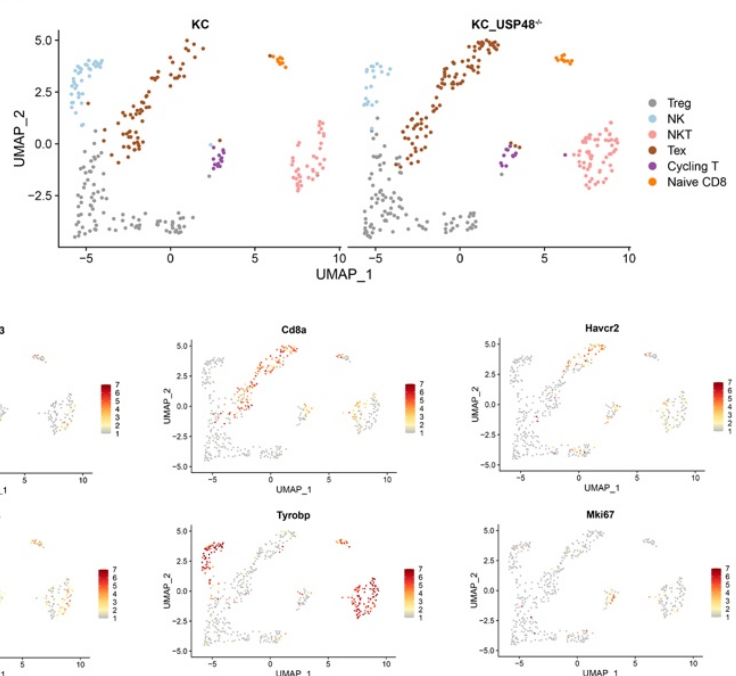

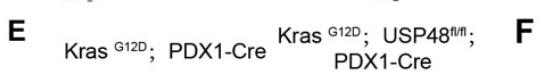

$F$

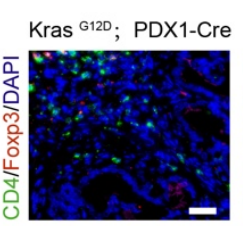
Kras ${ }^{\mathrm{G}}{ }^{12 \mathrm{D}}$; USP48 $8^{\mathrm{nN}}$;

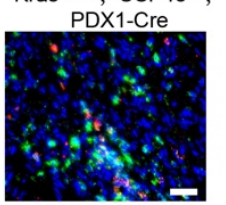

H

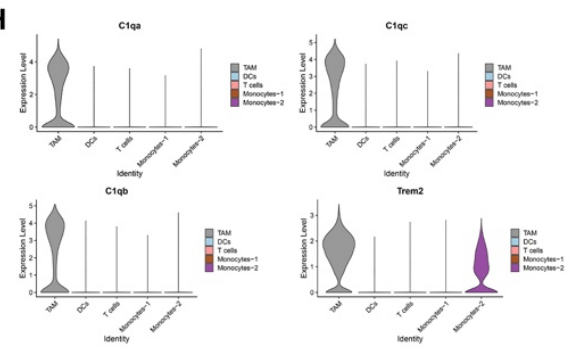

1

2

3

4

5

6

7

Figure 7. Knockout of USP48 increases the proportion of depleted T cells, Tregs and TAMs infiltrating pancreatic cancer.

(A) $\mathrm{KC}$ and $\mathrm{KC}$;USP48-/- pancreatic cancer tissue $\mathrm{T}$ cell subgroup analysis. (B) UMAP shows the difference between KC and KC;USP48-/- pancreatic cancer T cell subpopulation. (C) UMAP shows the standard marker gene for $\mathrm{T}$ cell subgroup classification. (D) Histogram shows the proportions of KC and KC;USP48-/-pancreatic cancer cells of tissue T cell subsets. (E) Multicolor fluorescent staining of $\mathrm{KC}$ and $\mathrm{KC}$;USP48-/- Tex cell levels in pancreatic cancer tissues. (F) Multicolor fluorescent staining of $\mathrm{KC}$ and $\mathrm{KC}$;USP48-/- Treg cells in pancreatic cancer tissues (horizontal). (G) UMAP showing KC and KC;USP48-/- TAMs in pancreatic cancer tissues. (H) Violin image showing specific marker genes in TAMs. (I) Multicolor fluorescent staining of $\mathrm{KC}$ and $\mathrm{KC}$;USP48${ }^{\text {- }}$ TAM cell levels in pancreatic cancer tissues; scale bar, $20 \mu \mathrm{m}$. 
Fig 8

A

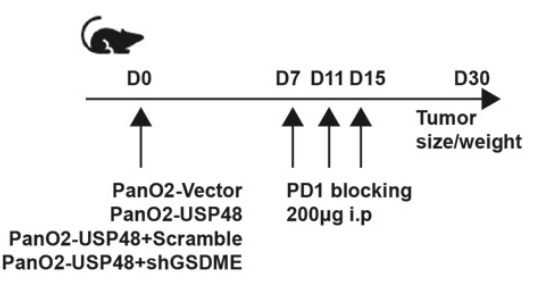

B

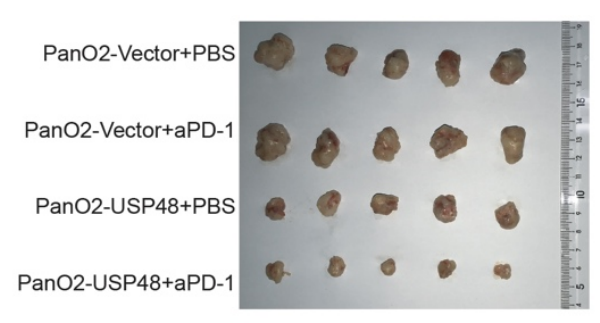

$\mathbf{F}$
C

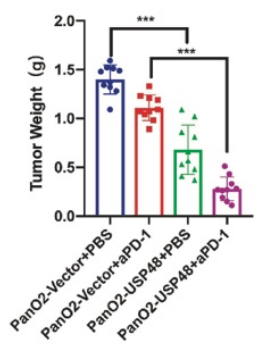

D

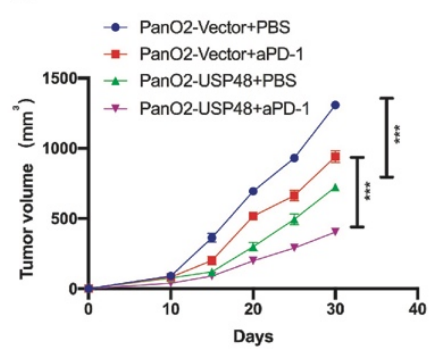

$E$

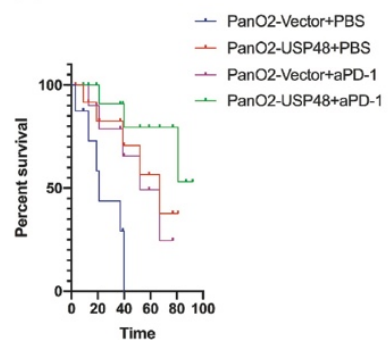

H

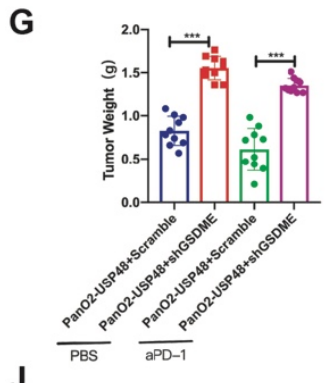

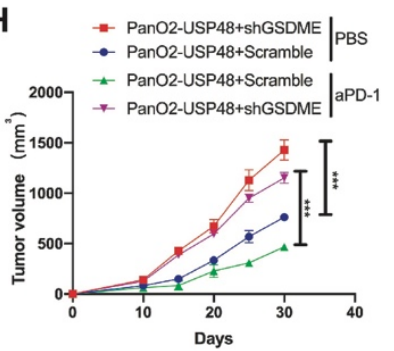

$\mathbf{J}$
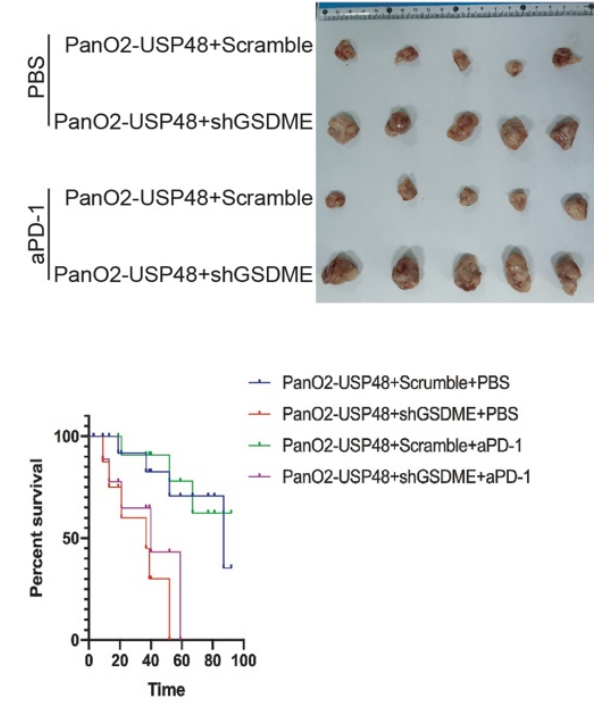
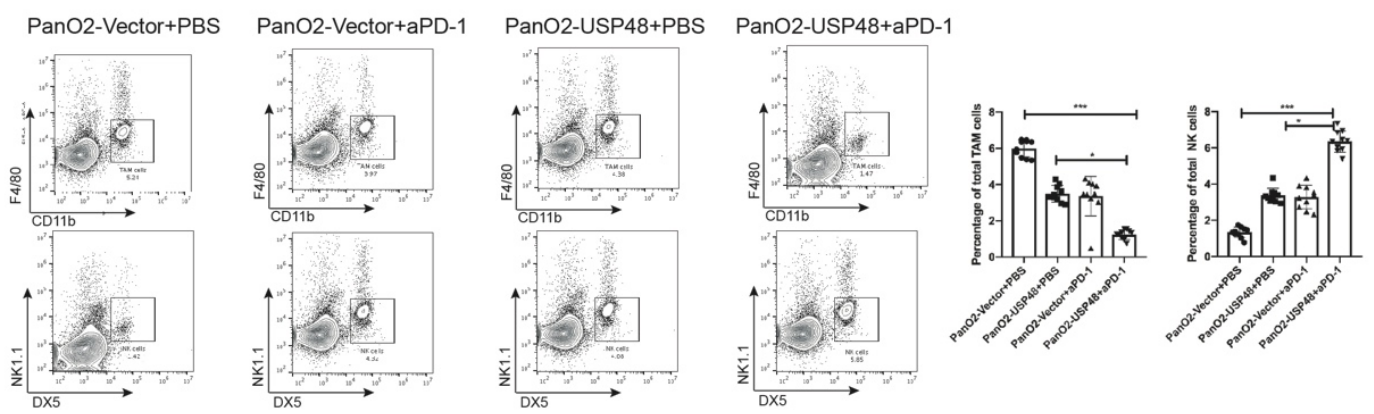

K

PanO2-USP48+Scramble PanO2-USP48+shGSDME

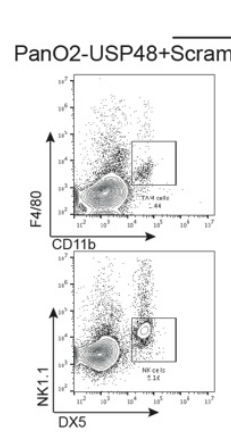

aPD-1
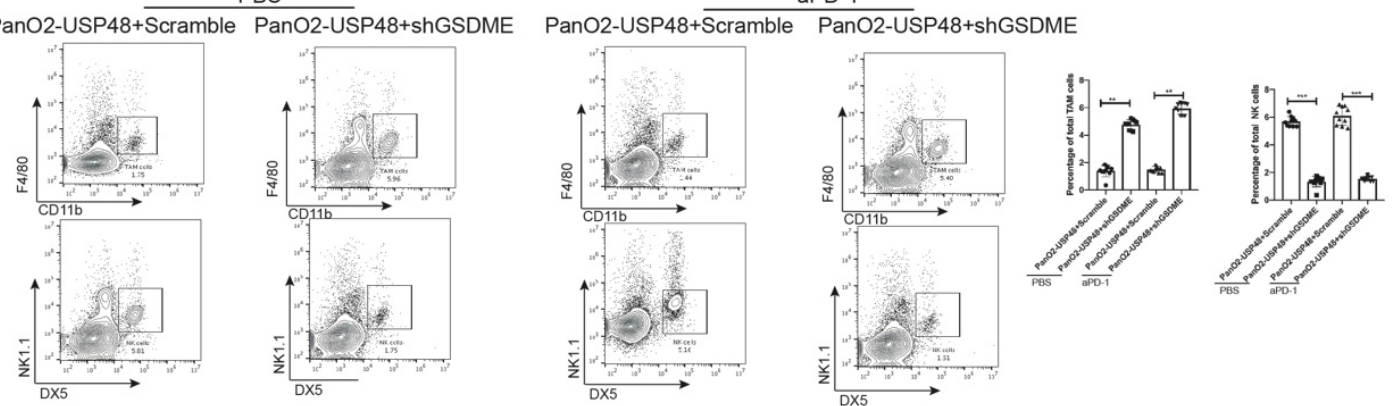

Figure 8. USP48-GSDME affects the sensitivity of mice to anti-PD-1 immunotherapy.

(A) Experimental flow chart. (B, F) Tumor photos after subcutaneous implantation of Pan02 cells under different treatments. $(\mathrm{C}, \mathrm{G})$ Tumor weight after 30 days of subcutaneous implantation of Pan02 cells under different treatments. (D, H) Volume changes of tumors within 30 days after subcutaneous implantation of Pan02 cells under different treatments. (E, I) Survival statistics of C57 mice after subcutaneous implantation of Pan02 cells under different treatments. ( $\mathrm{J}$ and $\mathrm{K}$ ) Flow 
1 cytometry assay to detect the proportion of TAMs in the tumor tissue. Data are shown as mean \pm 2 SD of three independent experiments. ${ }^{*} \mathrm{p}<0.05 ;{ }^{* *} \mathrm{p}<0.01 ; * * \mathrm{p}<0.001 ; * * * \mathrm{p}<0.0001 ;$ n.s., 3 not significant. 


\section{Supplementary Files}

This is a list of supplementary files associated with this preprint. Click to download.

- SupplementaryTable1.xlsx

- SupplementaryTable2.xlsx

- SupplementaryTable3.xlsx

- REAGENTorRESOURCE.pdf

- SupplementaryFigures.pdf 